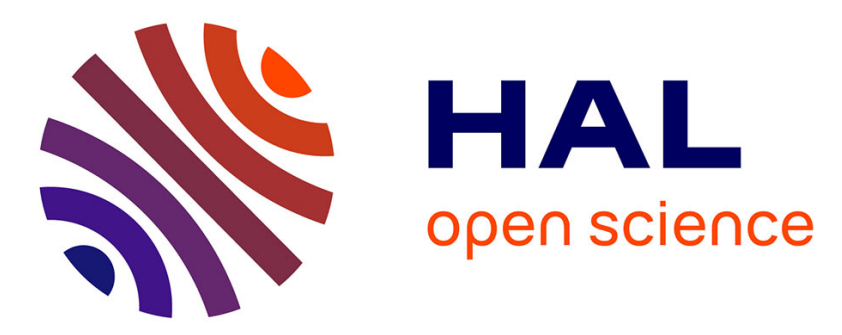

\title{
Renormalization group second order approximation for singularly perturbed nonlinear ordinary differential equations
}

Anna Marciniak-Czochra, Andro Mikelic, Thomas Stiehl

\section{- To cite this version:}

Anna Marciniak-Czochra, Andro Mikelic, Thomas Stiehl. Renormalization group second order approximation for singularly perturbed nonlinear ordinary differential equations. Mathematical Methods in the Applied Sciences, 2018, 41, pp.5691-5710. hal-01795884

\section{HAL Id: hal-01795884 \\ https://univ-lyon1.hal.science/hal-01795884}

Submitted on 18 May 2018

HAL is a multi-disciplinary open access archive for the deposit and dissemination of scientific research documents, whether they are published or not. The documents may come from teaching and research institutions in France or abroad, or from public or private research centers.
L'archive ouverte pluridisciplinaire HAL, est destinée au dépôt et à la diffusion de documents scientifiques de niveau recherche, publiés ou non, émanant des établissements d'enseignement et de recherche français ou étrangers, des laboratoires publics ou privés. 


\title{
Renormalization group second order approximation for singularly perturbed nonlinear ordinary differential equations
}

\author{
Anna Marciniak-Czochra \\ Institute of Applied Mathematics, IWR and BIOQUANT \\ University of Heidelberg \\ Im Neuenheimer Feld 267, 69120 Heidelberg , Germany
}

Andro Mikelić

Univ Lyon, Université Claude Bernard Lyon 1, CNRS UMR 5208,

Institut Camille Jordan, 43 blvd. du 11 novembre 1918, F-69622 Villeurbanne cedex, France

Thomas Stiehl

Institute of Applied Mathematics, IWR and BIOQUANT

University of Heidelberg

Im Neuenheimer Feld 267, 69120 Heidelberg , Germany

the article is accepted for publication in Mathematical Methods in the Applied Sciences, 2018

\begin{abstract}
We consider a two time scale nonlinear system of ordinary differential equations. The small parameter of the system is the ratio $\varepsilon$ of the scales. We search for an approximation involving only the slow time unknowns and valid uniformly for all times at order $O\left(\varepsilon^{2}\right)$. It is a classical problem, studied using the Tikhonov's singular perturbation theorem. We develop an approach leading to a higher order approximation using the renormalization group (RG) method. We apply it in two steps. In the first step we show that the RG method allows to approximate the fast time variables by their RG expansion taken at the slow time unknowns. Next we study the slow time equations, where the fast time unknowns are replaced by their RG expansion and show the second order uniform error estimate. The procedure is computationally less demanding than the classical Vasil'eva-O'Malley expansion and allows a higher order extension of Hoppensteadt's result on the Tikhonov singular perturbation theorem for infinite times.
\end{abstract}

\section{Introduction}

In 1994, Chen, Goldenfeld and Oono proposed a simple and unified method for treating asymptotic perturbation problems [4], [5]. This procedure originates from the quantum field theory and has been called the renormalization group (RG) method. In references [4] and [5] it is demonstrated that the RG method has several advantages compared with the conventional methods of multiscale expansion. One advantage of the RG method is that its starting point is a naive 
perturbation expansion. Therefore, special intuition or experience to guess the asymptotic expansion are not needed. The RG method applies to solving linear and nonlinear boundary layer problems. It is well-known that straightforward perturbation expansions lead to the appearance of secular terms, i.e., terms exhibiting unbounded growth in time. Using the RG method, these terms are renormalized and lead to the RG equation which yields correct asymptotic expansions. Unexpectedly, the RG method seems to automatically identify all scales relevant to the problem [4], [5]. Another remarkable property of the RG method is that it is sufficient to construct only the inner expansion. The RG method introduced in Ref. [4], [5] has been further developed in Ref. [18], [21] and studied rigorously in Ref. [10] and in a series of articles [6]-[9] by Chiba.

The mathematically rigorous results apply to systems of ordinary differential equations of the form

$$
\frac{d \mathbf{x}}{d t}=F \mathbf{x}+\varepsilon \mathbf{h}(\mathbf{x}, t)
$$

where $\varepsilon>0$ is a small parameter and $F$ is a matrix with purely imaginary eigenvalues. Treating this problem using the naive expansion approach results in secular terms and the obtained asymptotic expansions are not valid for time intervals of the length $T=O\left(\frac{1}{\varepsilon}\right)$. In contrast to the naive expansion approach, the RG method provides a good approximation also for long times as shown in [6]- [10].

We note a number of papers on the application of the $\mathrm{RG}$ method to partial differential equations and, in particular, to the geostrophic flows, see [22], [25], [28]. For a study of the shadow limit for systems of ODEs coupled with nonlinear parabolic equations, we refer to [20]. In the remainder of this paper we focus on systems of nonlinear ODEs.

We consider the asymptotic analysis of the ODE system with two characteristic times. Their ratio is equal to a small parameter $\varepsilon>0$. Motivated by biological applications, we focus on the Cauchy problem

$$
\begin{gathered}
\frac{d \mathbf{u}_{\varepsilon}}{d t}=\mathbf{f}\left(\mathbf{u}_{\varepsilon}, v_{\varepsilon}\right), t>0, \quad \mathbf{u}_{\varepsilon}(0)=\mathbf{u}^{0} \\
\varepsilon \frac{d v_{\varepsilon}}{d t}=-\alpha v_{\varepsilon}+\Phi\left(\mathbf{u}_{\varepsilon}, v_{\varepsilon}\right), t>0, \quad v_{\varepsilon}(0)=v^{0}
\end{gathered}
$$

The asymptotic analysis of problem (1)-(2) attracted a considerable interest in the literature. The convergence to the solutions of the reduced system

$$
-\alpha v+\Phi(\mathbf{u}, v)=0 \quad \text { and } \quad \frac{d \mathbf{u}}{d t}=\mathbf{f}(\mathbf{u}, v)
$$

was established for the stable roots $v=\varphi(\mathbf{u})$ in the seminal theorem by Tikhonov (see e.g., [2] and [26]). It holds for finite time intervals and its extension to the infinite time interval is due to Hoppensteadt [12]. In applications, it is important to derive an error estimate for the approximation. For finite time intervals error estimates at all orders are achieved through boundary layer corrections by Vasil'eva and colleges (see [27]). The work of Hoppensteadt [13] presents analogous estimates and also formulates, without proof, results for infinite time intervals. For a complete discussion of the subject we refer to a book by Hoppensteadt [14].

As observed by Chen, Goldenfeld and Oono in [5], the approach of Hoppensteadt [13], O'Malley [17] and Vasil'eva [27] requires simultaneous expansion in all equations and leads to complex and non-straightforward calculations. Unlike the classical approach, the RG method 
allows to apply the asymptotic expansion first to equation (2) and then, independently, to equation (1). After renormalization of the initial condition, a Cauchy problem for a single system of ODEs is obtained. With such choice of approximation, the RG method provides a uniform $O\left(\varepsilon^{2}\right)$ error estimate on $[T,+\infty], T=O(1)$. In addition, we construct effective initial conditions leading to a global $O\left(\varepsilon^{2}\right)$ estimate.

Another powerful technique for studying system (1)-(2) is the geometric singular perturbation theory (GSPT). The GSPT requires using normal forms. It applies to compact normally hyperbolic submanifolds of $F(u, v)=-\alpha v+\Phi(u, v)=0$, i.e., submanifolds consisting of roots of the nonlinear function $F$ in which the eigenvalues of the gradient of $F$ have non-zero real parts. For a detailed presentation of the method we refer to [15] and the references therein. The method has similarities to the Tikhonov theory but it is more general. The connection between RG and GSPT is discussed in [10]. It is shown that RG provides a systematic method for finding normal forms for large classes of finite-dimensional vector fields. It has advantages over the normal form theory, since it allows systematic identification of secular terms by inspection of naive asymptotic expansions.

In this paper, using the RG theory, we derive an $O\left(\varepsilon^{2}\right)$ approximation of (1)-(2) that involves only the variables of the slow equations (1). We obtain a Tikhonov type quasi-stationary approximation of order $\varepsilon^{2}$ for all times. Compared with the seminal work by Hoppensteadt [13] and his monograph [14] our results are new, since we provide a proof of the error estimate of order $\varepsilon^{2}$ on the infinite interval. Another advantage of our construction is in its simplicity compared to the boundary layers calculations in the classical approach of Vasil'eva. Our presentation is self-contained and accessible to non-specialists in dynamical systems.

The paper is organized as follows. We formally derive the RG equation in Section 2. In Section 3 we derive the effective equations of problem (1)-(2) using the center manifold approach. We notice that they are identical to the RG approximation from Section 2. Next, in Section 4 we introduce the setting in which the reduction to (3) is achieved at the order $O\left(\varepsilon^{2}\right)$ for all times. Our construction works for any isolated stable root $v$ of $-\alpha v+\Phi(\mathbf{u}, v)=0$. In Section 5 we present examples from the biosciences. The technical part of our results is postponed until Section 6.

\section{RG approach to the singular perturbation}

We study Cauchy's problem (1)-(2). The nonlinearities $\mathbf{f}$ and $\Phi$ are defined on $\mathbb{R}^{m+1}, m \geq 1$, and take values respectively in $\mathbb{R}^{m}$ and $\mathbb{R}^{1}$. It is assumed that they are $C^{2}$ with bounded derivatives and that problem (1)-(2) has a unique globally defined smooth solution. $\alpha$ is a positive constant.

In order to apply the renormalization group (RG) approach, we change the time scale by setting $\tau=t / \varepsilon$. System (1)-(2) becomes

$$
\frac{d \mathbf{u}_{\varepsilon}}{d \tau}=\varepsilon \mathbf{f}\left(\mathbf{u}_{\varepsilon}, v_{\varepsilon}\right), \frac{d v_{\varepsilon}}{d \tau}=-\alpha v_{\varepsilon}+\Phi\left(\mathbf{u}_{\varepsilon}, v_{\varepsilon}\right), \mathbf{u}_{\varepsilon}(0)=\mathbf{u}^{0}, v_{\varepsilon}(0)=v^{0} .
$$

We now proceed by the usual RG method steps.

1. We pretend that the problem can be solved as a regular perturbation problem and try a naive expansion

$$
\mathbf{u}_{\varepsilon}(\tau)=\mathbf{u}_{0}(\tau)+\varepsilon \mathbf{u}_{1}(\tau)+\varepsilon^{2} \mathbf{u}_{2}(\tau)+\ldots, \quad v_{\varepsilon}(\tau)=v_{0}(\tau)+\varepsilon v_{1}(\tau)+\ldots
$$


It gives

$$
\begin{gathered}
\frac{d}{d \tau}\left(\mathbf{u}_{0}(\tau)+\varepsilon \mathbf{u}_{1}(\tau)+\varepsilon^{2} \mathbf{u}_{2}(\tau)+\ldots\right)=\varepsilon \mathbf{f}\left(\mathbf{u}_{0}, v_{0}\right)+ \\
\varepsilon^{2}\left(\nabla_{u} \mathbf{f}\left(\mathbf{u}_{0}, v_{0}\right) \mathbf{u}_{1}(\tau)+\partial_{v} \mathbf{f}\left(\mathbf{u}_{0}, v_{0}\right) v_{1}(\tau)\right)+\ldots, \\
\frac{d}{d \tau}\left(v_{0}(\tau)+\varepsilon v_{1}(\tau)+\ldots\right)=-\alpha\left(v_{0}(\tau)+\varepsilon v_{1}(\tau)+\ldots\right)+\Phi\left(\mathbf{u}_{0}, v_{0}\right)+ \\
\varepsilon\left\{\nabla_{u} \Phi\left(\mathbf{u}_{0}, v_{0}\right) \mathbf{u}_{1}(\tau)+\frac{\partial \Phi}{\partial v}\left(\mathbf{u}_{0}, v_{0}\right) v_{1}(\tau)\right\}+\ldots
\end{gathered}
$$

We sort terms with respect to the powers of $\varepsilon$. Therefore, at order zero we have

$$
\frac{d}{d \tau} v_{0}(\tau)=-\alpha v_{0}+\Phi\left(\mathbf{u}_{0}(\tau), v_{0}(\tau)\right) \quad \text { and } \quad \frac{d}{d \tau} \mathbf{u}_{0}(\tau)=0 .
$$

We set

$$
\mathbf{u}_{0}(0)=\mathbf{A} \quad \text { and } \quad v_{0}(0)=B
$$

Then

$$
\mathbf{u}_{0}(\tau)=\mathbf{A} .
$$

In order to continue we have to make additional assumptions on $\Phi$.

First, we restrict ourselves to the case with $-\alpha v+\Phi(\mathbf{A}, v)=0$ having a unique solution. This assumption follows the Tikhonov theory and we make it here to streamline the presentation. The assumption will be relaxed in Section 4 .

Assumption 1 It holds

$$
-\kappa_{0} \leq-\xi:=-\alpha+\frac{\partial \Phi}{\partial v} \leq-\kappa<0
$$

in $\mathbb{R}^{m+1}$ for some positive constants $\kappa_{0}$ and $\kappa_{1}$.

We denote by $\varphi(\mathbf{A})$ the unique root of $-\alpha v+\Phi(\mathbf{A}, v)=0$.

Lemma 1 Under hypothesis (8) we have the estimate

$$
\left|v_{0}(\tau)-\varphi(\mathbf{A})\right| \leq C e^{-\kappa \tau},
$$

where $C$ and $\kappa$ are positive constants. Consequently

$$
v_{0}(\tau)=\varphi(\mathbf{A})+O\left(e^{-\kappa \tau}\right) .
$$

Proof. Denote by $\operatorname{sign}_{\mu}(\cdot), \mu \in \mathbb{R}$ regularizations of the sign function that converge to the sign function for $\mu$ passing to zero. Multiplying equation (5) by regularized sign $\left(v_{0}(\tau)-\varphi(\mathbf{A})\right)$ and passing to the zero limit of the regularization parameter $\mu$, we obtain the estimate $(9)$.

We now consider terms of order $O(\varepsilon)$ and obtain

$$
\begin{gathered}
\frac{d}{d \tau} v_{1}(\tau)=-\alpha v_{1}(\tau)+\nabla_{u} \Phi\left(\mathbf{A}, v_{0}\right) \mathbf{u}_{1}(\tau)+\frac{\partial \Phi}{\partial v}\left(\mathbf{A}, v_{0}\right) v_{1}(\tau), \\
\frac{d}{d \tau} \mathbf{u}_{1}(\tau)=\mathbf{f}\left(\mathbf{A}, v_{0}(\tau)\right) .
\end{gathered}
$$

In analogy to Lemma 1, we have: 
Dec11 Lemma 2 Under hypothesis (8) there is a constant $\mathbf{C}_{1 u}$ such that the following estimate holds

$$
\left|\mathbf{u}_{1}(\tau)-\tau \mathbf{f}(\mathbf{A}, \varphi(\mathbf{A}))-\mathbf{C}_{1 u}\right|+\left|\frac{d}{d \tau}\left(\mathbf{u}_{1}(\tau)-\tau \mathbf{f}(\mathbf{A}, \varphi(\mathbf{A}))\right)\right| \leq C e^{-\kappa \tau} .
$$

Consequently,

$$
\mathbf{u}_{1}(\tau)=\tau \mathbf{f}(\mathbf{A}, \varphi(\mathbf{A}))+\mathbf{C}_{1 u}+O\left(e^{-\kappa \tau}\right) .
$$

Proof. In analogy to Lemma 1.

It remains to calculate $v_{1}$ and $u_{2}$. We use equation (10) to calculate $v_{1}$. Using (9) and (12), we find out that equation (10) can be written as

$$
\frac{d}{d \tau} v_{1}(\tau)=-\xi^{A} v_{1}(\tau)+\nabla_{u} \Phi(\mathbf{A}, \varphi(\mathbf{A}))\left(\tau \mathbf{f}(\mathbf{A}, \varphi(\mathbf{A}))+\mathbf{C}_{1 u}\right)+\mathbf{F}_{1},
$$

where

$$
\xi^{A}=\alpha-\frac{\partial \Phi}{\partial v}(\mathbf{A}, \varphi(\mathbf{A})) \quad \text { and } \quad\left|\mathbf{F}_{1}(\tau)\right| \leq C e^{-\kappa_{1} \tau}, \kappa_{1}<\kappa .
$$

Note that $\xi^{A}$ is strictly positive. Therefore, we have

$$
\begin{gathered}
v_{1}(\tau)=v_{1}(0) \exp \left\{-\xi^{A} \tau\right\}+\nabla_{u} \Phi(\mathbf{A}, \varphi(\mathbf{A})) \cdot \int_{0}^{\tau} \exp \left\{-\xi^{A}(\tau-y)\right\}\left(\mathbf{C}_{1 u}\right. \\
+y \mathbf{f}(\mathbf{A}, \varphi(\mathbf{A}))) d y+\int_{0}^{\tau} \exp \left\{-\xi^{A}(\tau-y)\right\} \mathbf{F}_{1}(y) d y= \\
\nabla_{u} \Phi(\mathbf{A}, \varphi(\mathbf{A})) \cdot \frac{1}{\xi^{A}}\left(\tau \mathbf{f}(\mathbf{A}, \varphi(\mathbf{A}))+\mathbf{C}_{1 u}-\frac{\mathbf{f}(\mathbf{A}, \varphi(\mathbf{A}))}{\xi^{A}}\right)+O\left(e^{-\kappa_{1} \tau}\right), \kappa_{1}<\kappa .
\end{gathered}
$$

At order $O\left(\varepsilon^{2}\right)$ for $\mathbf{u}_{2}$ we have

$$
\frac{d}{d \tau} \mathbf{u}_{2}(\tau)=\nabla_{u} \mathbf{f}\left(\mathbf{u}_{0}, v_{0}\right) \mathbf{u}_{1}(\tau)+\partial_{v} \mathbf{f}\left(\mathbf{u}_{0}, v_{0}\right) v_{1}(\tau)
$$

Using (9), (12) and (14), we find out that equation (15) can be written as

$$
\begin{aligned}
& \frac{d}{d \tau} \mathbf{u}_{2}(\tau)=\nabla_{u} \mathbf{f}(\mathbf{A}, \varphi(\mathbf{A}))\left(\tau \mathbf{f}(\mathbf{A}, \varphi(\mathbf{A}))+\mathbf{C}_{1 u}\right)+\partial_{v} \mathbf{f}(\mathbf{A}, \varphi(\mathbf{A}))( \\
& \left.+\nabla_{u} \Phi(\mathbf{A}, \varphi(\mathbf{A})) \cdot \frac{1}{\xi^{A}}\left(\tau \mathbf{f}(\mathbf{A}, \varphi(\mathbf{A}))+\mathbf{C}_{1 u}-\frac{\mathbf{f}(\mathbf{A}, \varphi(\mathbf{A}))}{\xi^{A}}\right)\right)+\mathbf{F}_{2}
\end{aligned}
$$

where $\left|\mathbf{F}_{2}(\tau)\right| \leq C e^{-\kappa_{1} \tau}, \kappa_{1}<\kappa$. Therefore, we have

$$
\begin{gathered}
\mathbf{u}_{2}(\tau)=\frac{\tau^{2}}{2}\left(\nabla_{u} \mathbf{f}(\mathbf{A}, \varphi(\mathbf{A})) \mathbf{f}(\mathbf{A}, \varphi(\mathbf{A}))+\right. \\
\left.\partial_{v} \mathbf{f}(\mathbf{A}, \varphi(\mathbf{A})) \nabla_{u} \Phi(\mathbf{A}, \varphi(\mathbf{A})) \cdot \frac{1}{\xi^{A}} \mathbf{f}(\mathbf{A}, \varphi(\mathbf{A}))\right)+\tau\left(\nabla_{u} \mathbf{f}(\mathbf{A}, \varphi(\mathbf{A})) \mathbf{C}_{1 u}+\right. \\
\left.\partial_{v} \mathbf{f}(\mathbf{A}, \varphi(\mathbf{A})) \nabla_{u} \Phi(\mathbf{A}, \varphi(\mathbf{A})) \cdot \frac{1}{\xi^{A}}\left(\mathbf{C}_{1 u}-\frac{\mathbf{f}(\mathbf{A}, \varphi(\mathbf{A}))}{\xi^{A}}\right)\right)+\mathbf{C}_{2 u}+O\left(e^{-\kappa_{1} \tau}\right), \kappa_{1}<\kappa,
\end{gathered}
$$


where $\mathbf{C}_{2 u}$ is an arbitrary constant vector.

The approximation is now

$$
\begin{gathered}
\mathbf{u}_{\varepsilon}(\tau)=\mathbf{u}_{0}(\tau)+\varepsilon \mathbf{u}_{1}(\tau)+\varepsilon^{2} \mathbf{u}_{2}(\tau)+O\left(\varepsilon^{3}\right), \\
v_{\varepsilon}(\tau)=v_{0}(\tau)+\varepsilon v_{1}(\tau)+O\left(\varepsilon^{2}\right) .
\end{gathered}
$$

In most case, we detect immediately the presence of secular terms in $\mathbf{u}_{1}$. They can also appear in $\mathbf{u}_{2}$ and $v_{1}$.

2. The idea of the normalization is to introduce an arbitrary time $\mu$, split $\tau$ as $\tau-\mu+\mu$ and absorb the terms containing $\mu$ into the renormalized counterpart $\mathbf{A}(\mu)$ of $\mathbf{A}$. We introduce the renormalization constant $\mathbf{Z}^{1}=1+\mathbf{a}_{1} \varepsilon+\mathbf{a}_{2} \varepsilon^{2}$. The coefficient $\mathbf{A}$ is replaced by $\left(1+a_{1 k} \varepsilon+a_{2 k} \varepsilon^{2}\right) A_{k}(\mu), k=1, \ldots, m$. The coefficients $\mathbf{a}_{2}$ and $\mathbf{a}_{1}$ are chosen to eliminate the terms containing $\mu$ at order $O\left(\varepsilon^{2}\right)$.

Our approximation (18)-(19) becomes

$$
\begin{gathered}
u^{k}(\tau)=u_{0 k}(\tau)+\varepsilon u_{1 k}(\tau)+\varepsilon^{2} u_{2 k}(\tau)=\left(1+a_{1 k} \varepsilon+a_{2 k} \varepsilon^{2}\right) A_{k}(\mu)+ \\
\left.\varepsilon(\tau-\mu+\mu) f_{k}\left(\mathbf{A}(\mu)\left(1+\mathbf{a}_{1} \varepsilon\right)\right), \varphi\left(\mathbf{A}(\mu)\left(1+\mathbf{a}_{1} \varepsilon\right)\right)\right)+\varepsilon C_{1 u, k}+\varepsilon^{2} u_{2 k}(\tau), \\
v=v_{0}(\mu)+\varepsilon v_{1}(\mu)=\varphi\left(\mathbf{A}(\mu)\left(1+\mathbf{a}_{1} \varepsilon\right)\right)+ \\
\varepsilon \nabla_{u} \Phi(\mathbf{A}, \varphi(\mathbf{A})) \cdot \frac{1}{\xi^{A}}\left((\tau-\mu+\mu) \mathbf{f}(\mathbf{A}, \varphi(\mathbf{A}))+\mathbf{C}_{1 u}-\frac{\mathbf{f}(\mathbf{A}, \varphi(\mathbf{A}))}{\xi^{A}}\right) .
\end{gathered}
$$

We choose $a_{1 k}$ such that the term $\mu f_{k}\left(\mathbf{Z}^{1} \mathbf{A}, \varphi\left(\mathbf{Z}^{1} \mathbf{A}\right)\right)$ is eliminated at the leading order. Hence we have

$$
a_{1 k}(\mu) A_{k}(\mu)+\mu f_{k}(\mathbf{A}, \varphi(\mathbf{A}))+C_{1 u, k}=0,
$$

implying

$$
a_{1 k}=-\frac{\mu}{A_{k}} f_{k}(\mathbf{A}, \varphi(\mathbf{A}))-\frac{C_{1 u, k}}{A_{k}}, \quad k=1, \ldots, m .
$$

Therefore, for $u_{1}$ we have the following expression

$$
\begin{gathered}
\left.\left.\mathbf{u}_{1}(\tau)=(\tau-\mu) \mathbf{f}\left(\left(1+\mathbf{a}_{1} \varepsilon\right)\right) \mathbf{A}, \varphi\left(\left(1+\mathbf{a}_{1} \varepsilon\right)\right) \mathbf{A}\right)\right)+R=(\tau-\mu) \mathbf{f}(\mathbf{A}, \varphi(\mathbf{A})) \\
-\varepsilon(\tau-\mu)\left(\nabla_{u} \mathbf{f}(\mathbf{A}, \varphi(\mathbf{A})) \mathbf{C}_{1 u}+\partial_{v} \mathbf{f}(\mathbf{A}, \varphi(\mathbf{A})) \nabla_{A} \varphi(\mathbf{A}) \cdot \mathbf{C}_{1 u}\right)- \\
\varepsilon(\tau-\mu) \mu\left(\nabla_{u} \mathbf{f}(\mathbf{A}, \varphi(\mathbf{A})) \mathbf{f}(\mathbf{A}, \varphi(\mathbf{A}))+\frac{\partial_{v} \mathbf{f}(\mathbf{A}, \varphi(\mathbf{A}))}{\xi^{A}} \nabla_{u} \Phi(\mathbf{A}, \varphi(\mathbf{A})) \cdot \mathbf{f}(\mathbf{A}, \varphi(\mathbf{A}))\right) \\
+R,
\end{gathered}
$$

where $R$ and its derivative with respect to $\tau$ are exponentially small in $\tau$.

Next, expressions (14) and (22) yield

$$
\begin{gathered}
v_{0}(\mu)+\varepsilon v_{1}(\mu)=\varphi(\mathbf{A})+\varepsilon\left(\nabla_{A} \varphi(\mathbf{A}) \cdot \mathbf{a}_{1} \mathbf{A}++\right. \\
\left.\nabla_{u} \Phi(\mathbf{A}, \varphi(\mathbf{A})) \cdot \frac{1}{\xi^{A}}\left((\tau-\mu+\mu) \mathbf{f}(\mathbf{A}, \varphi(\mathbf{A}))+\mathbf{C}_{1 u}-\frac{\mathbf{f}(\mathbf{A}, \varphi(\mathbf{A}))}{\xi^{A}}\right)\right)= \\
\varphi(\mathbf{A})+\varepsilon\left(\tau-\mu-\frac{1}{\xi^{A}}\right) \nabla_{u} \Phi(\mathbf{A}, \varphi(\mathbf{A})) \cdot \frac{\mathbf{f}(\mathbf{A}, \varphi(\mathbf{A}))}{\xi^{A}}
\end{gathered}
$$


We now transform $\tau$ into $\tau-\mu+\mu$ and $\tau^{2}$ into $\tau^{2}-\mu^{2}+\mu^{2}$ in the expression (17) for $\mathbf{u}_{2}(\mu)$. Inserting formulas (23) and renormalized (17) into (20) yields $u^{k}(\tau)$ without secular terms in $\mu$. The terms of order $O\left(\varepsilon^{2}\right)$, containing only $\mu$ and $\mu^{2}$, are to be eliminated and only terms containing $\tau-\mu$ and $\tau^{2}-\mu^{2}$ remain. We achieve this goal by choosing appropriate $a_{2 k}$. After recalling that

$$
\nabla \varphi(\mathbf{A})\left(\alpha-\frac{\partial \Phi}{\partial v}(\mathbf{A}, \varphi(\mathbf{A}))=\nabla_{u} \Phi(\mathbf{A}, \varphi(\mathbf{A})),\right.
$$

we obtain the following expression for $\mathbf{u}(\tau)$ :

$$
\begin{gathered}
\mathbf{u}(\tau)=\mathbf{A}(\mu)+\varepsilon(\tau-\mu) \mathbf{f}(\mathbf{A}, \varphi(\mathbf{A}))-\varepsilon^{2}(\tau-\mu) \frac{\nabla_{u} \Phi(\mathbf{A}, \varphi(\mathbf{A})) \cdot \mathbf{f}(\mathbf{A}, \varphi(\mathbf{A}))}{\left(\xi^{A}\right)^{2}} \partial_{v} \mathbf{f}(\mathbf{A}, \varphi(\mathbf{A})) \\
+\frac{\varepsilon^{2}}{2}(\tau-\mu)^{2}\left(\nabla_{u} \mathbf{f}(\mathbf{A}, \varphi(\mathbf{A})) \mathbf{f}(\mathbf{A}, \varphi(\mathbf{A}))+\right. \\
\left.\frac{\partial_{v} \mathbf{f}(\mathbf{A}, \varphi(\mathbf{A}))}{\xi^{A}} \nabla_{u} \Phi(\mathbf{A}, \varphi(\mathbf{A})) \cdot \mathbf{f}(\mathbf{A}, \varphi(\mathbf{A}))\right) .
\end{gathered}
$$

3. The parameter $\mu$ is artificial and the solution does not depend on it. Therefore, it has to hold $\left.\frac{\partial \mathbf{u}(\tau)}{\partial \mu}\right|_{\mu=\tau}=0$ for all $\tau$. After noticing that terms multiplying $\varepsilon^{2} \mu$ cancel, $\left.\frac{\partial \mathbf{u}(\tau)}{\partial \mu}\right|_{\mu=\tau}=$ 0 implies the RG equation

$$
\frac{d \mathbf{A}}{d \tau}=\varepsilon \mathbf{f}(A, \varphi(A))-\varepsilon^{2} \frac{\nabla_{u} \Phi(\mathbf{A}, \varphi(\mathbf{A})) \cdot \mathbf{f}(\mathbf{A}, \varphi(\mathbf{A}))}{\left(\alpha-\frac{\partial \Phi}{\partial v}(\mathbf{A}, \varphi(\mathbf{A}))\right)^{2}} \partial_{v} \mathbf{f}(\mathbf{A}, \varphi(\mathbf{A})) .
$$

Returning to the original variable $t=\varepsilon \tau$, we obtain

$$
\frac{d \mathbf{A}}{d t}=\mathbf{f}(A, \varphi(A))-\varepsilon \frac{\nabla_{u} \Phi(\mathbf{A}, \varphi(\mathbf{A})) \cdot \mathbf{f}(\mathbf{A}, \varphi(\mathbf{A}))}{\left(\alpha-\frac{\partial \Phi}{\partial v}(\mathbf{A}, \varphi(\mathbf{A}))\right)^{2}} \partial_{v} \mathbf{f}(\mathbf{A}, \varphi(\mathbf{A})),
$$

which is the RG equation we were looking for. For $t=O(1)$ the initial time layer effects became negligible and the approximation is expressed by $\mathbf{A}(t)$.

4. It remains to check the value of the derivative of $v(\tau)=v_{0}(\mu)+\varepsilon v_{1}(\mu)$ with respect to $\mu$ at $\mu=\tau$. A direct calculation yields

$$
\begin{gathered}
\left.\frac{\partial v(\tau)}{\partial \mu}\right|_{\mu=\tau}=\nabla \varphi(\mathbf{A}) \frac{d \mathbf{A}}{d \tau}-\varepsilon \frac{\mathbf{f}(A, \varphi(A)) \cdot \nabla_{u} \Phi(A, \varphi(A))}{\alpha-\frac{\partial \Phi}{\partial v}(\mathbf{A}, \varphi(\mathbf{A}))}- \\
\varepsilon \frac{d}{d \mu}\left(\frac{\mathbf{f}(A, \varphi(A)) \cdot \nabla_{u} \Phi(A, \varphi(A))}{\left(\alpha-\frac{\partial \Phi}{\partial v}(\mathbf{A}, \varphi(\mathbf{A}))\right)^{2}}\right)=O\left(\varepsilon^{2}\right) .
\end{gathered}
$$

Therefore, the choice

$$
\begin{gathered}
\mathbf{u}_{\varepsilon}(t) \approx \mathbf{A}^{R G}(t), \\
v_{\varepsilon}(t) \approx \varphi\left(\mathbf{A}^{R G}(t)\right)-\varepsilon \frac{\mathbf{f}\left(\mathbf{A}^{R G}, \varphi\left(\mathbf{A}^{R G}\right)\right) \cdot \nabla_{u} \Phi\left(\mathbf{A}^{R G}, \varphi\left(\mathbf{A}^{R G}\right)\right)}{\left(\alpha \mathbf{A}^{R G}-\Phi\left(\mathbf{A}^{R G}, \varphi\left(\mathbf{A}^{R G}\right)\right)\right)^{2}},
\end{gathered}
$$

where $\mathbf{A}^{R G}$ is expressed by (27), provides the requested $O\left(\varepsilon^{2}\right)$ approximation, for times $O(1) \leq t \leq O(1 / \varepsilon)$. The correct behavior for small times is described by the initial time layers, as in the classical literature (see e.g., [17], [26] and [27]). 


\section{Center Manifold Theorem approach to obtain the effective model}

In this section we present a formal derivation of the effective equations using the Center Manifold Theorem.

The usual way to study the qualitative behavior of the solutions of the equation

$$
\frac{d \mathbf{y}}{d t}=\Psi(\mathbf{y}), \quad \Psi(0)=0,
$$

is to calculate the Jacobian matrix $A=\left.\nabla \Psi\right|_{y=0}$ and to study the linearized problem

$$
\frac{d \mathbf{y}}{d t}=A \mathbf{y}+\mathbf{G}(\mathbf{y}), \quad \mathbf{G}(0)=0, \nabla \mathbf{G}(0)=0 .
$$

If all the eigenvalues of the matrix $A=\left.\nabla \Psi\right|_{y=0}$ have non-zero real part, then the HartmanGrobman theorem applies and the behavior of equation (30) in a small neighborhood of $\mathbf{0}$ is described by equation (31).

If there exist eigenvalues with zero real part, a center manifold $W^{c}$ is spanned by the corresponding (generalized) eigenvectors. In this case the Center Manifold Theorem is used to restrict the analysis to such a "center manifold". For an introduction to the center manifold technique, we quote the classical book by Carr [3].

Our goal is to study behavior of the solution to problem (1)-(2), namely

$$
\begin{gathered}
\frac{d \mathbf{u}_{\varepsilon}}{d t}=\mathbf{f}\left(\mathbf{u}_{\varepsilon}, v_{\varepsilon}\right), t>0, \quad \mathbf{u}_{\varepsilon}(0)=\mathbf{u}^{0} \\
\frac{d v_{\varepsilon}}{d t}=-\frac{\alpha}{\varepsilon} v_{\varepsilon}+\frac{1}{\varepsilon} \Phi\left(\mathbf{u}_{\varepsilon}, v_{\varepsilon}\right), t>0, \quad v_{\varepsilon}(0)=v^{0},
\end{gathered}
$$

for $\varepsilon \rightarrow 0$.

We notice immediately the large difference between the modulus of the eigenvalues related to equation (2) and those related to equation (1). Therefore, it is to be expected that equation (1) will play the role of the center manifold for problem (1)-(2).

More precisely, the solution for (2) is given by

$$
v_{\varepsilon}-\frac{1}{\alpha} \Phi\left(\mathbf{u}_{\varepsilon}, v_{\varepsilon}\right)=v^{0} e^{-\alpha t / \varepsilon}+\left(\frac{1}{\varepsilon} e^{-\alpha t / \varepsilon}-\frac{1}{\alpha} \delta(t)\right) * \Phi\left(\mathbf{u}_{\varepsilon}, v_{\varepsilon}\right),
$$

where $*$ denotes the convolution product in time and $\delta(t)$ the delta distribution centered at $t$. Under mild assumptions on $\Phi$, the right hand side converges to zero, as $\varepsilon \rightarrow 0$. We conclude that $v_{\varepsilon} \approx \frac{1}{\alpha} \Phi\left(\mathbf{u}_{\varepsilon}, v_{\varepsilon}\right)$, for small $\varepsilon$.

Hence, it is reasonable to assume that $v$ lies on an invariant manifold, which we represent as $v=Z\left(\mathbf{u}_{\varepsilon}\right)$. On the invariant manifold it holds

$$
\frac{d v}{d t}=\frac{d}{d t} Z(\mathbf{u})=\nabla Z \frac{d}{d t} \mathbf{u}, \quad \forall t .
$$

We substitute the above identity into (2) and obtain

$$
-\alpha Z(\mathbf{u})+\Phi(\mathbf{u}, Z(\mathbf{u}))=\varepsilon \nabla Z \cdot \mathbf{f}(\mathbf{u}, Z(\mathbf{u})) .
$$


We expand $Z$ as

$$
Z=Z_{0}+\varepsilon Z_{1}+O\left(\varepsilon^{2}\right)
$$

Sorting terms with respect to their orders of $\varepsilon$, we obtain

$$
\begin{gathered}
O(1): \quad-\alpha Z_{0}+\Phi\left(\mathbf{u}, Z_{0}\right)=0 \\
O(\varepsilon): \quad-\alpha Z_{1}+\partial_{v} \Phi\left(\mathbf{u}, Z_{0}\right) Z_{1}=\nabla Z_{0} \cdot \mathbf{f}\left(\mathbf{u}, Z_{0}\right) .
\end{gathered}
$$

The first equation implies that $Z_{0}$ is a root of $v-\frac{1}{\alpha} \Phi(\mathbf{u}, v)=0$. The second equation implies that

$$
Z_{1}=\frac{\nabla Z_{0} \cdot \mathbf{f}\left(\mathbf{u}, Z_{0}\right)}{-\alpha+\partial_{v} \Phi\left(\mathbf{u}, Z_{0}\right)}
$$

Hence

$$
v=Z_{0}+\varepsilon \frac{\nabla Z_{0} \cdot \mathbf{f}\left(\mathbf{u}, Z_{0}\right)}{-\alpha+\partial_{v} \Phi\left(\mathbf{u}, Z_{0}\right)}
$$

To obtain the ODE for $\mathbf{u}$, we substitute expression (34) into (1):

$$
\begin{gathered}
\frac{d \mathbf{u}}{d t}=\mathbf{f}\left(\mathbf{u}, \varphi(\mathbf{u})-\varepsilon \frac{\nabla_{u} \Phi(\mathbf{u}, \varphi(\mathbf{u})) \cdot \mathbf{f}(\mathbf{u}, \varphi(\mathbf{u}))}{\left(\alpha-\frac{\partial \Phi}{\partial v}(\mathbf{u}, \varphi(\mathbf{u}))\right)^{2}}\right)= \\
\mathbf{f}(\mathbf{u}, \varphi(\mathbf{u}))-\varepsilon \frac{\nabla_{u} \Phi(\mathbf{u}, \varphi(\mathbf{u})) \cdot \mathbf{f}(\mathbf{u}, \varphi(\mathbf{u}))}{\left(\alpha-\frac{\partial \Phi}{\partial v}(\mathbf{u}, \varphi(\mathbf{u}))\right)^{2}} \partial_{v} \mathbf{f}(\mathbf{u}, \varphi(\mathbf{u}))+O\left(\varepsilon^{2}\right) .
\end{gathered}
$$

We note that equation (35) is identical to equation (27).

Remark 1 It is possible to transform the above intuitive argument into a rigorous one. Nevertheless, most results obtained using the Center Manifold Theorem describe dynamics of small solutions around the origin. In our problem we would like to prove that for small values of the parameter $\varepsilon$ we remain for all times in an $\varepsilon^{k}$ - neighborhood of the solution of equation (27). The reduction to a neighborhood of zero is not straightforward and we prefer presenting a direct proof, based on the computations from Section 2 and the boundary layers, adopted from Tikhonov's theory.

\section{Main results}

We start by making basic assumptions on our singularly perturbed system.

Assumption 2 Let $(\mathbf{f}, \Phi) \in C^{3}\left(\mathbb{R}^{m+1}\right)^{m+1}, m \geq 1$, and let the algebraic equation

$$
-\alpha y+\Phi(\mathbf{x}, y)=0 \quad \text { in } \quad \mathbb{R},
$$

have at least one isolated real root $y=y(\mathbf{x})$.

Assumption 3 We suppose that the reduced problem

$$
-\alpha v+\Phi(\mathbf{A}, v)=0 ; \quad \frac{d \mathbf{A}}{d t}=\mathbf{f}(\mathbf{A}, v), t>0, \quad \text { and } \quad \mathbf{A}(0)=\mathbf{u}^{0},
$$

Reduced1

has a smooth bounded solution $\{\mathbf{A}, v\}$ on $\mathbb{R}_{+}$and that $v$ is an isolated real root. 
Ass3 Assumption 4 We suppose that the chosen root $v$ satisfies

$$
\xi=\alpha-\left.\partial_{y} \Phi(\mathbf{x}, y)\right|_{(\mathbf{x}=\mathbf{A}, y=v)} \geq M_{0}>0 \quad \text { on } \quad \mathbb{R}_{+},
$$

i.e., that $v$ is a stable root.

In addition, we suppose that the initial datum $v^{0}$ is in the basin of attraction of the root $v$ from (37) (i.e., for all $\delta>0$, there exists $t(\delta)>0$ such that the solution $w$ of the initial value problem $\frac{d}{d t} w=-\alpha w+\Phi(\mathbf{A}(t), w), w(0)=v^{0}$ satisfies $|w(t)-\varphi(\mathbf{A}(t))| \leq \delta$ for $\left.t>t(\delta)\right)$. We furthermore assume that on the interval $\mathcal{I}=\left[\min \left\{\varphi\left(\mathbf{u}^{0}\right), v^{0}\right\}, \max \left\{\varphi\left(\mathbf{u}^{0}\right), v^{0}\right\}\right]$ we have

$$
\alpha-\left.\partial_{y} \Phi(\mathbf{x}, y)\right|_{\left(\mathbf{x}=\mathbf{u}^{0}, y \in \mathcal{I}\right)} \geq \frac{M_{0}}{2}>0 .
$$

Next, we introduce the initial layer $\zeta_{0}$ by

$$
\begin{gathered}
\frac{d \zeta_{0}}{d \tau}=-\alpha \zeta_{0}+\Phi\left(\mathbf{u}^{0}, \varphi\left(\mathbf{u}^{0}\right)+\zeta_{0}(\tau)\right)-\Phi\left(\mathbf{u}^{0}, \varphi\left(\mathbf{u}^{0}\right)\right), \\
\zeta_{0}(0)=v^{0}-\varphi\left(\mathbf{u}^{0}\right)
\end{gathered}
$$

$\operatorname{Inn} 2 \mathrm{pbz}$

(to be compared with (9)). Note that by Assumption 4, $\varphi\left(\mathbf{u}^{0}\right)$ is uniquely defined and applying Lemma 1 implies

$$
\left|\zeta_{0}(\tau)\right| \leq C e^{-M_{0} \tau}
$$

Note that $\zeta_{0}(t / \varepsilon)$ is the classical initial layer function from [13], [17] and [27].

For an error estimate another assumption is needed:

Ass4 Assumption 5 We suppose that the matrix

$$
\left.\left(\nabla_{u} \mathbf{f}(\mathbf{x}, \varphi(x))+\partial_{v} \mathbf{f}(\mathbf{x}, \varphi(x)) \otimes \nabla \varphi(\mathbf{x})\right)\right|_{\mathbf{x}=\mathbf{A}}
$$

satisfies the exponential dichotomy assumption on $\mathbb{R}_{+}$, i.e., we assume that a fundamental solution $S$ of the linear system corresponding to (42) fulfills $S(0)=I,\left\|S(t) S^{-1}(\tau)\right\| \leq K e^{-\kappa(t-s)}$ for $s \leq t \leq \infty$ and appropriate positive constants $K, \kappa$.

Dichotomy Remark 2 We recall that this is a special case of the exponential dichotomy assumption, stating that there exists a projection $P$, such that $\left\|S(t) P S^{-1}(\tau)\right\| \leq K e^{-\kappa(t-s)}$ for $s \leq t \leq \infty$ and $\left\|S(t)(I-P) S^{-1}(\tau)\right\| \leq L e^{\mu(t-s)}$ for $s \geq t \geq-\infty$ for appropriate positive constants $K, L, \kappa, \mu$, [13], [16].

Remark2 Remark 3 Let $b>0$ and

$$
\mathcal{T}_{b, \lambda}=\operatorname{Int} \cup_{t \in \mathbb{R}_{+}}\{\mathbf{A}(t)+\mathbf{x}, \varphi(\mathbf{A})+y\}_{\left\{\left|x_{j}\right|<b, j=1, \ldots, m, y \in I_{\lambda}(t)\right\}},
$$

where $\lambda(t)$ is a smooth curve, defined on $\mathbb{R}_{+}$and such that $\lambda(0)=\left|\varphi\left(\mathbf{u}^{0}\right)-v^{0}\right|$ and $\lambda(t)=\lambda_{0}>0$ for $t \geq t_{0}$. The set $I_{\lambda}$ is given by

$$
I_{\lambda}(t)= \begin{cases}(-b, \lambda(t)), & \text { for } v^{0} \geq \varphi\left(\mathbf{u}^{0}\right), \\ (-\lambda(t), b), & \text { for } v^{0}<\varphi\left(\mathbf{u}^{0}\right) .\end{cases}
$$

Since $\mathbf{A}$ is bounded and continuous, and because of (39) in Assumption 4, there exists a tubular domain $\mathcal{T}_{b, \lambda}$ defined by (43) and depicted in Fig. 1, such that (38) and (42) hold true in $\mathcal{T}_{b, \lambda}$. Note that the existence of exponential dichotomies of the considered form is preserved under small continuous perturbations or continuous perturbations vanishing as $t \rightarrow \infty$, see [16] or [1] respectively. Note that for all times $\left|\zeta_{0}(t / \varepsilon)\right| \leq \lambda(t)$. 

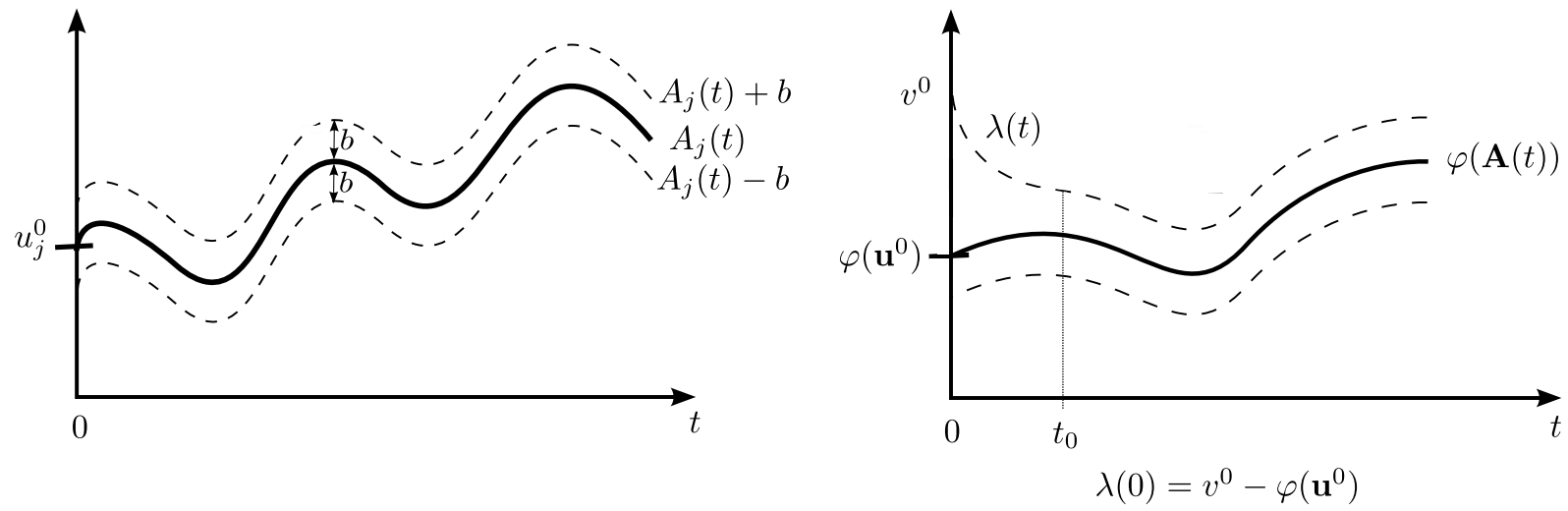

Figure 1: The tubular domain $\mathcal{T}_{b, \lambda}$.

fig:tube

We now define $C^{\infty}$-cutoff extensions of the nonlinearities $\mathbf{f}$ and $\Phi$. Let $\xi \in \mathbb{R}$ and $g$ a $C^{\infty}$-function such that

$$
g(\xi)= \begin{cases}\xi, & \text { for }|\xi| \leq 1 / 2 \\ 1, & \text { for } \xi \geq 1 \\ -1, & \text { for } \xi \leq-1\end{cases}
$$

smoothy

Then we replace $\Phi$ by $\tilde{\Phi}$, where

$$
\tilde{\Phi}\left(x_{1}, \ldots, x_{m+1}, t\right)=\Phi\left(\tilde{x}_{1}, \ldots, \tilde{x}_{m+1}\right), \quad\left(x_{1}, \ldots, x_{m+1}, t\right) \in \mathbb{R}^{m+1} \times \mathbb{R}_{+},
$$

and

$$
\tilde{x}_{j}=g\left(\frac{x_{j}-A_{j}(t)}{b}\right) b+A_{j}(t)
$$

for $j=1, \ldots, m$ and

$$
\tilde{x}_{m+1}=g\left(\frac{x_{m+1}-\varphi(\mathbf{A}(t))}{\lambda(t)}\right) \lambda(t)+\varphi(\mathbf{A}(t)) .
$$

The function $\tilde{\Phi}$ is smooth, globally Lipschitz and satisfies condition (38) for $(\mathbf{x}, y) \in \mathbb{R}^{m+1}$ and $t \in \mathbb{R}_{+}$.

We replace $\mathbf{f}$ by $\tilde{\mathbf{f}}$, where

$$
\tilde{\mathbf{f}}\left(x_{1}, \ldots, x_{m+1}, t\right)=\mathbf{f}\left(\tilde{x}_{1}, \ldots, \tilde{x}_{m+1}\right), \quad\left(x_{1}, \ldots, x_{m+1}, t\right) \in \mathbb{R}^{m+1} \times \mathbb{R}_{+},
$$

and $\left(\tilde{x}_{1}, \ldots, \tilde{x}_{m+1}\right)$ as defined in (47)-(48). The function $\tilde{\mathbf{f}}$ is smooth, globally Lipschitz and satisfies condition (42) for $(\mathbf{x}, y) \in \mathbb{R}^{m+1}$ and $t \in \mathbb{R}_{+}$. Therefore, the problem

$$
\begin{gathered}
\frac{d \mathbf{u}_{\varepsilon}}{d t}=\tilde{\mathbf{f}}\left(\mathbf{u}_{\varepsilon}, v_{\varepsilon}, t\right), t>0, \quad \mathbf{u}_{\varepsilon}(0)=\mathbf{u}^{0} \\
\varepsilon \frac{d v_{\varepsilon}}{d t}=-\alpha v_{\varepsilon}+\tilde{\Phi}\left(\mathbf{u}_{\varepsilon}, v_{\varepsilon}, t\right), t>0, \quad v_{\varepsilon}(0)=v^{0} .
\end{gathered}
$$

has a unique $C^{1,1}$ solution on $[0, T]$, for all $0<T<+\infty$. Furthermore, the $C^{1,1}$ norm is uniformly bounded.

Remark 4 The above $C^{\infty}$ - cutoff extension of the nonlinearities is motivated by the similar extensions in the center manifold theory. For more details we refer to the seminal book of Carr [3], p.16-17. 
We study the error function for equation (51) and define

$$
\mathcal{V}_{\varepsilon}(t):=v_{\varepsilon}(t)-\tilde{\varphi}\left(\mathbf{u}_{\varepsilon}(t), t\right)-\tilde{\zeta}_{0}\left(\frac{t}{\varepsilon}\right), t>0,
$$

where $\tilde{\varphi}$ is the modification of the root $\varphi$ of equation (36), obtained after replacing $\Phi$ by $\tilde{\Phi}$ and $\tilde{\zeta}_{0}$ is the solution of problem (40) obtained after replacing $\Phi$ by $\tilde{\Phi}$.

Prop0 Proposition 1

$$
\left|\mathcal{V}_{\varepsilon}(t)\right| \leq C \varepsilon, \quad 0 \leq t
$$

where $C$ does not depend on the extension.

Proof. See Section 6.2.

Next, we study the error function for $\mathbf{u}_{\varepsilon}$ and define

$$
\delta_{\varepsilon}(t):=\mathbf{u}_{\varepsilon}(t)-\mathbf{A}(t), t \geq 0 .
$$

Under the above assumptions, the error function $\delta_{\varepsilon}$ satisfies the following Cauchy problem

$$
\begin{gathered}
\frac{d \delta_{\varepsilon}}{d t}=\tilde{\mathbf{f}}\left(\mathbf{A}+\delta_{\varepsilon}, \varphi\left(\mathbf{A}+\delta_{\varepsilon}, t\right)+\zeta_{0}\left(\frac{t}{\varepsilon}\right)+\mathcal{V}_{\varepsilon}, t\right)-\tilde{\mathbf{f}}(\mathbf{A}, \varphi(\mathbf{A}), t), t>0 \\
\delta_{\varepsilon}(0)=0 .
\end{gathered}
$$

Errorder1

Tikh1G

The following result holds:

Prop2 Proposition 2 Problem (55)-(56) has a unique solution $\delta_{\varepsilon}$ satisfying

$$
\left|\delta_{\varepsilon}(t)\right| \leq C \varepsilon, \quad 0 \leq t,
$$

where $C$ does not depend on the extension and the time interval, furthermore $C \leq \lambda(0)$.

Proof. See Section 6.2.

Cor1 Corollary 1 For $\varepsilon \leq \varepsilon_{0}$ it holds $\tilde{\mathbf{f}}=\mathbf{f}$ and $\tilde{\Phi}=\Phi$ in a neighborhood of the solution. Hence the solutions of problem (50)-(51) coincide with the solution of problem (1)-(2). They exist for all times.

Remark 5 In [12] the proof that the solution of problem (1)-(2) remains in the tubular neighborhood $\mathcal{T}_{b, \lambda}$ is carried out using a local Lyapunov functional. In our setting, this property results from the error estimate.

longtime Remark 6 Let us replace the exponential dichotomy from Assumption 5 by the assumption that the matrix

$\left.\left(\nabla_{u} \mathbf{f}(\mathbf{x}, \varphi(x))+\partial_{v} \mathbf{f}(\mathbf{x}, \varphi(x)) \otimes \nabla \varphi(\mathbf{x})\right)\right|_{\mathbf{x}=\mathbf{A}} \quad$ has a symmetric part with non-positive eigenvalues.

Then for every $\gamma>0$,

Stabil1L

$$
\left|\delta_{\varepsilon}(t)\right| \leq C \varepsilon^{1-\gamma}, \quad 0 \leq t \leq C \varepsilon^{-\gamma},
$$

where $C$ does not depend on the extension and the time interval. 
Proof. See Section 6.2.

Th1 Theorem 1 Under Assumptions 2-5, it holds

$$
\begin{gathered}
\sup _{0 \leq t \leq+\infty}\left|\mathbf{u}_{\varepsilon}(t)-\mathbf{A}(t)\right| \leq C \varepsilon, \\
\left|v_{\varepsilon}(t)-\varphi(\mathbf{A}(t))\right| \leq C\left(\varepsilon+e^{-M_{0} t / \varepsilon}\right), \quad \forall t \in \mathbb{R}_{+} .
\end{gathered}
$$

Proof. The statement follows from Propositions 1 and 2.

Prop3 Proposition 3 Let

$$
\mathcal{U}_{\varepsilon}=v_{\varepsilon}-\varphi\left(\mathbf{u}_{\varepsilon}\right)+\frac{\varepsilon}{\alpha-\frac{\partial \Phi}{\partial v}\left(\mathbf{u}_{\varepsilon}, \varphi\left(\mathbf{u}_{\varepsilon}\right)\right)} \frac{d}{d t} \varphi\left(\mathbf{u}_{\varepsilon}\right)-\zeta_{0}(t / \varepsilon)-\varepsilon \zeta_{1}(t / \varepsilon),
$$

where $\zeta_{1}$ fulfills

$$
\frac{d}{d t} \zeta_{1}(t)=-\alpha \zeta_{1}(t), \zeta_{1}(0)=\frac{\nabla \varphi\left(\mathbf{u}^{\mathbf{0}}\right)}{\alpha-\frac{\partial \Phi}{\partial v}\left(\mathbf{u}^{\mathbf{0}}, \varphi\left(\mathbf{u}^{\mathbf{0}}\right)\right)} \mathbf{f}\left(\mathbf{u}^{\mathbf{0}}, v^{0}\right) .
$$

Under Assumptions 2-5, it holds

$$
\mathcal{U}_{\varepsilon}(t)=\mathcal{U}_{\varepsilon, 1}(t)+\mathcal{U}_{\varepsilon, 2}(t)
$$

where $\mathcal{U}_{\varepsilon, 1}$ are $\mathcal{U}_{\varepsilon, 2}$ are differentiable functions fulfilling

$$
\left\|\mathcal{U}_{\varepsilon, 1}\right\|_{L^{\infty}\left(\mathbb{R}_{+}\right)} \leq C \varepsilon^{2}, \quad\left\|\mathcal{U}_{\varepsilon, 2}\right\|_{L^{1}\left(\mathbb{R}_{+}\right)} \leq C \varepsilon^{2} .
$$

Proof. See Section 6.2.

Prop4 Proposition 4 Let Assumptions 2-5 be satisfied. Define $\hat{\delta}_{\varepsilon}(t)=\mathbf{u}_{\varepsilon}(t)-\mathbf{A}(t)-\varepsilon \mathbf{u}^{1}(t)-\varepsilon \psi^{1}(t / \varepsilon)$ with

$$
\left\{\begin{array}{l}
\frac{d}{d \tau} \psi^{1}=\mathbf{f}\left(\mathbf{u}^{0}, \varphi\left(\mathbf{u}^{0}\right)+\zeta_{0}(\tau)\right)-\mathbf{f}\left(\mathbf{u}^{0}, \varphi\left(\mathbf{u}^{0}\right)\right) \\
\psi^{1}(0)=-\int_{0}^{\infty}\left(\mathbf{f}\left(\mathbf{u}^{0}, \varphi\left(\mathbf{u}^{0}\right)+\zeta_{0}(\tau)\right)-\mathbf{f}\left(\mathbf{u}^{0}, \varphi\left(\mathbf{u}^{0}\right)\right)\right) d \tau
\end{array}\right.
$$

and

$$
\left\{\begin{aligned}
\frac{d}{d t} \mathbf{u}^{1}= & \left.\left(\nabla_{u} \mathbf{f}(\mathbf{x}, y)+\partial_{v} \mathbf{f}(\mathbf{x}, y) \otimes \nabla \varphi(\mathbf{x})\right)\right|_{(\mathbf{x}=\mathbf{A}, y=\varphi(\mathbf{A}))} \mathbf{u}^{1} \\
& -\partial_{v} \mathbf{f}(\mathbf{A}, \varphi(\mathbf{A})) \frac{\nabla \varphi(\mathbf{A}) \mathbf{f}(\mathbf{A}, \varphi(\mathbf{A}))}{\alpha-\frac{\partial \Phi}{\partial v}(\mathbf{A}, \varphi(\mathbf{A}))} \\
\mathbf{u}^{1}(0)= & -\psi^{1}(0) .
\end{aligned}\right.
$$

Then, the following estimate holds for all $t \geq 0$ :

$$
\left|\hat{\delta}_{\varepsilon}(t)\right| \leq C \varepsilon^{2} .
$$

Proof. See Section 6.2. 
Theorem 2 Let Assumptions 2-5 be satisfied. For $\varepsilon$ small enough it holds

(i) $\sup _{T_{0} \leq t \leq+\infty}\left|\mathbf{u}_{\varepsilon}(t)-\mathbf{A}^{R G}(t)\right| \leq C \varepsilon^{2}, \quad T_{0}=\mathcal{O}(1)$

(ii) $\sup _{T_{0} \leq t \leq+\infty}\left|v_{\varepsilon}(t)-\varphi\left(\mathbf{A}^{R G}\right)+\frac{\varepsilon}{\xi\left(\mathbf{A}^{R G}\right)} \nabla \varphi\left(\mathbf{A}^{R G}\right) \cdot \mathbf{f}\left(\mathbf{A}^{R G}, \varphi\left(\mathbf{A}^{R G}\right)\right)\right| \leq C \varepsilon^{2}, \quad T_{0}=\mathcal{O}(1)$, where $\mathbf{A}^{R G}$ fulfills the 1st order RG equation

$$
\left\{\begin{array}{l}
\frac{d}{d t} \mathbf{A}^{R G}=\mathbf{f}\left(\mathbf{A}^{R G}, \varphi\left(\mathbf{A}^{R G}\right)\right) \\
-\frac{\varepsilon}{\xi\left(\mathbf{A}^{R G}\right)} \nabla \varphi\left(\mathbf{A}^{R G}\right) \cdot \mathbf{f}\left(\mathbf{A}^{R G}, \varphi\left(\mathbf{A}^{R G}\right)\right) \partial_{v} \mathbf{f}\left(\mathbf{A}^{R G}, \varphi\left(\mathbf{A}^{R G}\right)\right) \\
\mathbf{A}^{R G}(0)=\mathbf{u}^{0}+\varepsilon \int_{0}^{\infty}\left(\mathbf{f}\left(\mathbf{u}^{0}, \varphi\left(\mathbf{u}^{0}\right)+\zeta_{0}(\tau)\right)-\mathbf{f}\left(\mathbf{u}^{0}, \varphi\left(\mathbf{u}^{0}\right)\right)\right) d \tau
\end{array}\right.
$$

Proof. First we observe that

$$
\mathbf{u}_{\varepsilon}(t)-\mathbf{A}^{R G}=u_{\varepsilon}(t)-\mathbf{A}(t)-\varepsilon \mathbf{u}^{1}(t)-\varepsilon \psi^{1}\left(\frac{t}{\varepsilon}\right)-\left(\mathbf{A}^{R G}-\mathbf{A}-\varepsilon \mathbf{u}^{1}\right)+\varepsilon \psi^{1}\left(\frac{t}{\varepsilon}\right) .
$$

Proposition 4 yields that $\hat{\delta}_{\varepsilon}=u_{\varepsilon}(t)-\mathbf{A}-\varepsilon \mathbf{u}^{1}-\varepsilon \psi^{1}\left(\frac{t}{\varepsilon}\right)$ is globally $O\left(\varepsilon^{2}\right)$ in $L^{\infty}$. Since $\varepsilon \psi^{1}\left(\frac{t}{\varepsilon}\right)$ decays exponentially, it is enough to estimate the error function $\mathbf{d}^{\varepsilon}:=\mathbf{A}^{R G}-\mathbf{A}-\varepsilon \mathbf{u}^{1}$. Using the ODEs for $\mathbf{A}^{R G}, \mathbf{A}$ and $\varepsilon \mathbf{u}^{1}$, we obtain

$$
\begin{gathered}
\frac{d}{d t} \mathbf{d}^{\varepsilon}(t)=\left.\left(\nabla_{u} \mathbf{f}(\mathbf{x}, y)+\partial_{v} \mathbf{f}(\mathbf{x}, y) \otimes \nabla \varphi(\mathbf{x})\right)\right|_{\left(\mathbf{x}=\mathbf{A}^{*}, y=\varphi\left(\mathbf{A}^{*}\right)\right)} \mathbf{d}^{\varepsilon}+\varepsilon^{2} \mathbf{G}^{*} \\
\text { on }(0,+\infty), \\
\mathbf{d}^{\varepsilon}(0)=0,
\end{gathered}
$$

where $\mathbf{G}^{*}$ is bounded. Error estimate (i) now follows directly.

In order to prove (ii), we use Proposition 3 and the estimate (i).

\section{Example of an application in biology}

We consider the following system of ordinary differential equations

$$
\begin{gathered}
\frac{d}{d t} u_{\varepsilon, 1}=\left(2 a v_{\varepsilon}-1\right) p u_{\varepsilon, 1}, \quad u_{\varepsilon, 1}(0)=u_{1}^{0}, \\
\frac{d}{d t} u_{\varepsilon, 2}=2\left(1-a v_{\varepsilon}\right) p u_{\varepsilon, 1}-d u_{\varepsilon, 2}, \quad u_{\varepsilon, 2}(0)=u_{2}^{0}, \\
\varepsilon \frac{d}{d t} v_{\varepsilon}=1-k u_{\varepsilon, 2} v_{\varepsilon}-v_{\varepsilon}, \quad v_{\varepsilon}(0)=v^{0},
\end{gathered}
$$

where $p, k, d$ are positive constants, $a \in(0.5,1)$ and $u_{1}^{0}, u_{2}^{0}, v^{0}$ are positive. This system describes time evolution of a cell system maintained by a stem cell population that differentiates to mature cells, e.g., blood cells [19], [24]. Then $v_{\varepsilon}$ is interpreted as the concentration of signaling molecules and $u_{\varepsilon, 1}, u_{\varepsilon, 2}$ as counts of stem and committed cells. The existence of (locally) stable positive equilibria and the dynamics in the neighborhood of such equilibria are of biological interest [23], [24]. The 0th-order RG approximation ("quasi steady state approximation") of this model 


$$
\begin{gathered}
\frac{d}{d t} A_{1}=\left(\frac{2 a}{1+k A_{2}}-1\right) p A_{1}, \quad A_{1}(0)=u_{1}^{0} \\
\frac{d}{d t} A_{2}=2\left(1-\frac{a}{1+k A_{2}}\right) p A_{1}-d A_{2}, \quad A_{2}(0)=u_{2}^{0} \\
v=\frac{1}{1+k A_{2}}
\end{gathered}
$$

has been extensively studied in references [11], [23] and [24], motivated by the different time scales inherent to the biological system. Our results provide a rigorous framework to show that in proximity of positive equilibria the distance between the solutions of the reduced system (73)-(74) and the original system (70)-(71) is bounded by $C \varepsilon$, and the distance of the solutions of the original system (70)-(71) and the first order RG-approximation is bounded by $C \varepsilon^{2}$ for times $t \geq \mathcal{O}(1)$.

Lemma 3 Consider system (70)-(72) and the corresponding 0th-order RG-approximation (73)(74). Both systems have a unique positive equilibrium. In the vicinity of this equilibrium Assumptions 2-5 are satisfied.

Proof. We have $\alpha=1, \Phi(\mathbf{x}, y)=-k x_{2} y+1, \mathbf{f}(\mathbf{x}, y)=\left[(2 a y-1) p x_{1}, 2(1-a y) p x_{1}-d x_{2}\right]^{T}$. Assumption 2 is trivially fulfilled with the isolated root $y(\mathbf{x})=\varphi(\mathbf{x})=\frac{1}{1+k x_{2}}$. We note that $\mathbf{A}$ is nonnegative for nonnegative initial conditions. Assumption 4 is fulfilled, since $\alpha-$ $\left.\partial_{y} \Phi(\mathbf{x}, y)\right|_{\mathbf{x}=\mathbf{A}, y=\varphi(\mathbf{A})}=1+k A_{2} \geq 1$. To show the boundedness of $\mathbf{A}$, we consider $q:=\frac{A_{1}}{A_{2}}$, which fulfills the initial value problem $q(0)=u_{1}^{0} / u_{2}^{0} \frac{d}{d t} q=\left(2 \frac{a}{1+k A_{2}}-1\right) p q-2\left(1-\frac{a}{1+k A_{2}}\right) p q^{2}+d q$ $\leq(2 a-1) p q-2(1-a) p q^{2}+d q$. Consequently, $q>\frac{(2 a-1) p+d_{2}}{2(1-a) p}=: Q$ implies $\frac{d}{d t} q<0$. Therefore, $q \leq \max \{Q, q(0)\}=: K_{1}$. Then we obtain $\frac{d}{d t} A_{1} \leq\left(\frac{2 a}{1+k A_{1} / K_{1}}-1\right) p A_{1}$. Therefore, $A_{1}>\frac{(2 a-1) K_{1}}{k}$ implies $\frac{d}{d t} A_{1}<0$. Consequently, $A_{1} \leq \max \left\{u_{1}^{0}, \frac{(2 a-1) K_{1}}{k}\right\}=: K_{2}$. This yields $\frac{d}{d t} A_{2} \leq 2 p K_{2}-d A_{2}$. Therefore, $A_{2} \leq \max \left\{u_{2}^{0}, \frac{2 p K_{2}}{d}\right\}$. Local Lipschitz continuity and global boundedness imply existence of global solutions, Assumption 3 is thus satisfied. The positive equilibrium is given by $\bar{y}=\frac{1}{1+k \bar{u}_{\varepsilon, 2}}, \bar{A}_{1}=\bar{u}_{\varepsilon, 1}=\frac{(2 a-1) d}{k p}, \bar{A}_{2}=\bar{u}_{\varepsilon, 2}=\frac{2 a-1}{k}$. The linearization of system (73)-(74) around the positive equilibrium has two eigenvalues with negative real parts, due to Vieta's theorem. Therefore, Assumption 5 is fulfilled for the constant solution $\mathbf{A}=\overline{\mathbf{A}}$. Since $\overline{\mathbf{A}}$ is locally stable it follows that solutions $\mathbf{A}$ of system (73)-(74) with initial values in the vicinity of $\overline{\mathbf{A}}$ converge exponentially to $\overline{\mathbf{A}}$. Due to stability of exponential dichotomies with respect to small continuous perturbations, Assumption 5 is fulfilled along trajectories of such solutions A.

The 1st-order RG approximation is given by the following Lemma. 
Lemma 4 The 1 st-order RG approximation of (70)-(72) is given by

$$
\begin{gathered}
\frac{d}{d t} A_{1}^{R G}=\left(\frac{2 a}{1+k A_{2}^{R G}}-1\right) p A_{1}^{R G} \\
+2 a p A_{1}^{R G} k \frac{2\left(1-\frac{a}{1+k A_{2}^{R G}}\right) p A_{1}^{R G}-d A_{2}^{R G}}{\left(1+k A_{2}^{R G}\right)^{3}} \varepsilon \\
\frac{d}{d t} A_{2}^{R G}=\left[2\left(1-\frac{a}{1+k A_{2}^{R G}}\right) p A_{1}^{R G}-d A_{2}^{R G}\right]\left(1-\frac{2 a p A_{1}^{R G} k}{\left(1+k A_{2}^{R G}\right)^{3}} \varepsilon\right) \\
\mathbf{A}^{R G}(0)=\left[u_{1}^{0}, u_{2}^{0}\right]^{T}+2 a p u_{1}^{0} \frac{\left(v^{0}-\frac{1}{1+k u_{2}^{0}}\right)}{1+k u_{2}^{0}}[1,-1]^{T} \varepsilon .
\end{gathered}
$$

Proof. We use the expressions for $\alpha, \Phi, \mathbf{f}, \varphi$ specified in the proof of Lemma 3, together with $\varphi(\mathbf{u})=\frac{1}{1+k u_{2}}, \nabla \varphi(\mathbf{u})=\left[0, \frac{-k}{\left(1+k u_{2}\right)^{2}}\right]^{T}, \partial_{v} \mathbf{f}(\mathbf{u}, v)=2 a p u_{1}[1,-1]^{T}$. The ODEs for $A_{1}^{R G}$ and $A_{2}^{R G}$ follow from Theorem 2.

For the initial layer $\zeta_{0}$ we obtain $\zeta_{0}(0)=v^{0}-\frac{1}{1+k u_{2}^{0}}, \frac{d}{d t} \zeta_{0}=-\left(1+k u_{2}^{0}\right) \zeta_{0}$ and therefore $\zeta_{0}(t)=\left(v^{0}-\frac{1}{1+k u_{2}^{0}}\right) e^{-\left(1+k u_{2}^{0}\right) t}$.

Furthermore, it holds

$$
\mathbf{f}\left(\mathbf{u}^{0}, \varphi\left(\mathbf{u}^{0}\right)+\zeta_{0}\right)-\mathbf{f}\left(\mathbf{u}^{0}, \varphi\left(\mathbf{u}^{0}\right)\right)=2 a \zeta_{0} p u_{1}^{0}[1,-1]^{T}
$$

and

$$
\int_{0}^{\infty}\left(\mathbf{f}\left(\mathbf{u}^{0}, \varphi\left(\mathbf{u}^{0}\right)+\zeta_{0}\right)-\mathbf{f}\left(\mathbf{u}^{0}, \varphi\left(\mathbf{u}^{0}\right)\right)\right) d t=\frac{\left(v^{0}-\frac{1}{1+k u_{2}^{0}}\right)}{1+k u_{2}^{0}}\left[2 a p u_{1}^{0},-2 a p u_{1}^{0}\right]^{T} .
$$

This yields the initial condition. Lemma 3 and Theorem 2 imply that the distance of solutions of the original system (70)-(71) and the first order RG-approximation (75)-(77) is bounded by $C \varepsilon^{2}$ for times $t \geq \mathcal{O}(1)$.

\section{Proof of the main results}

\subsection{Proof of Proposition 1}

Proof. In order to simplify notation, we systematically skip tilde overbars in the proof.

By definition $\mathcal{V}_{\varepsilon}(0)=0$ and

$$
\begin{gathered}
\varepsilon \frac{d}{d t} \mathcal{V}_{\varepsilon}=-\alpha v_{\varepsilon}+\Phi\left(\mathbf{u}_{\varepsilon}, v_{\varepsilon}, t\right)-\varepsilon \frac{d}{d t} \varphi\left(\mathbf{u}_{\varepsilon}, t\right)+\alpha \zeta_{0}(t / \varepsilon)+\Phi\left(\mathbf{u}^{0}, \varphi\left(\mathbf{u}^{0}, t\right), t\right) \\
-\Phi\left(\mathbf{u}^{0}, \varphi\left(\mathbf{u}^{0}, t\right)+\zeta_{0}(t / \varepsilon), t\right) \\
=-\alpha \mathcal{V}_{\varepsilon}+\Phi\left(\mathbf{u}_{\varepsilon}, v_{\varepsilon}, t\right)-\Phi\left(\mathbf{u}_{\varepsilon}, \varphi\left(\mathbf{u}_{\varepsilon}, t\right)+\zeta_{0}\left(\frac{t}{\varepsilon}\right), t\right)-\varepsilon \frac{d}{d t} \varphi\left(\mathbf{u}_{\varepsilon}, t\right) \\
+\alpha\left(\varphi\left(\mathbf{u}^{0}, t\right)+\zeta_{0}\left(\frac{t}{\varepsilon}\right)\right)+\Phi\left(\mathbf{u}_{\varepsilon}, \varphi\left(\mathbf{u}_{\varepsilon}, t\right)+\zeta_{0}(t / \varepsilon), t\right) \\
-\alpha\left(\varphi\left(\mathbf{u}_{\varepsilon}, t\right)+\zeta_{0}\left(\frac{t}{\varepsilon}\right)\right)-\Phi\left(\mathbf{u}^{0}, \varphi\left(\mathbf{u}^{0}, t\right)+\zeta_{0}\left(\frac{t}{\varepsilon}\right), t\right) \\
=-\left(\alpha-\frac{\partial \Phi^{\star}}{\partial v}\right) \mathcal{V}_{\varepsilon}-\varepsilon \frac{d}{d t} \varphi\left(\mathbf{u}_{\varepsilon}, t\right)+I(t)
\end{gathered}
$$


with

$$
\begin{gathered}
I(t):=\Phi\left(\mathbf{u}_{\varepsilon}, \varphi\left(\mathbf{u}_{\varepsilon}, t\right)+\zeta_{0}(t / \varepsilon), t\right)-\alpha\left(\varphi\left(\mathbf{u}_{\varepsilon}, t\right)+\zeta_{0}(t / \varepsilon)\right) \\
-\Phi\left(\mathbf{u}^{\mathbf{0}}, \varphi\left(\mathbf{u}^{\mathbf{0}}, t\right)+\zeta_{0}(t / \varepsilon), t\right)+\alpha\left(\varphi\left(\mathbf{u}^{\mathbf{0}}, t\right)+\zeta_{0}(t / \varepsilon)\right)
\end{gathered}
$$

and $\frac{\partial \Phi^{\star}}{\partial v}:=\frac{\partial \Phi\left(\mathbf{u}_{\varepsilon}, v^{\star}\right)}{\partial v}$, where $v^{\star}$ is an intermediate value between $v_{\varepsilon}$ and $\varphi\left(u_{\varepsilon}\right)$. In the above calculations we used equation $\alpha \varphi(\mathbf{x})=\Phi(\mathbf{x}, \varphi(\mathbf{x}, t), t)$ for all $\mathbf{x} \in \mathbb{R}^{m}$.

Next, we use the smoothness of the extensions and estimate $I(t)$ using the second order derivatives:

$$
\begin{gathered}
|I(t)|=\left|\int_{0}^{1} \frac{d}{d \eta}\left[\Phi\left(\mathbf{u}_{\varepsilon}(\eta t), \varphi\left(\mathbf{u}_{\varepsilon}(\eta t)\right)+\zeta_{0}(t / \varepsilon)\right)-\alpha\left(\varphi\left(\mathbf{u}_{\varepsilon}(\eta t)\right)+\zeta_{0}(t / \varepsilon)\right)\right] d \eta\right| \\
=\left.\left|\int_{0}^{1} t \nabla_{u} \Phi(\mathbf{u}, v)\right|_{\left.\mathbf{u}=\mathbf{u}_{\varepsilon}(\eta t), v=\varphi\left(\mathbf{u}_{\varepsilon}(\eta t)\right)+\zeta_{0}(t / \varepsilon)\right)} \cdot \frac{d}{d \tau} \mathbf{u}_{\varepsilon}(\tau)\right|_{\tau=\eta t} \\
\quad-\left.\left.t \nabla_{u} \Phi(\mathbf{u}, v)\right|_{\mathbf{u}=\mathbf{u}_{\varepsilon}(\eta t), v=\varphi\left(\mathbf{u}_{\varepsilon}(\eta t)\right)} \cdot \frac{d}{d \tau} \mathbf{u}_{\varepsilon}(\tau)\right|_{\tau=\eta t} \\
+\left.\left.t \partial_{v} \Phi(\mathbf{u}, v)\right|_{\left.\mathbf{u}=\mathbf{u}_{\varepsilon}(\eta t), v=\varphi\left(\mathbf{u}_{\varepsilon}(\eta t)\right)+\zeta_{0}(t / \varepsilon)\right)} \nabla \varphi\left(\mathbf{u}_{\varepsilon}(\eta t)\right) \cdot \frac{d}{d \tau} \mathbf{u}_{\varepsilon}(\tau)\right|_{\tau=\eta t} \\
-\left.\left.t \partial_{v} \Phi(\mathbf{u}, v)\right|_{\mathbf{u}=\mathbf{u}_{\varepsilon}(\eta t), v=\varphi\left(\mathbf{u}_{\varepsilon}(\eta t)\right)} \nabla \varphi\left(\mathbf{u}_{\varepsilon}(\eta t)\right) \cdot \frac{d}{d \tau} \mathbf{u}_{\varepsilon}(\tau)\right|_{\tau=\eta t} \\
-\left.t \alpha \nabla \varphi\left(\mathbf{u}_{\varepsilon}(\eta t)\right) \cdot \frac{d}{d \tau} \mathbf{u}_{\varepsilon}(\tau)\right|_{\tau=\eta t}+\left.t \alpha \nabla \varphi\left(\mathbf{u}_{\varepsilon}(\eta t)\right) \cdot \frac{d}{d \tau} \mathbf{u}_{\varepsilon}(\tau)\right|_{\tau=\eta t} d \eta \mid \\
\leq t C \int_{0}^{1}\left|\zeta_{0}(t / \varepsilon)\right| d \eta \leq t C e^{-M_{0} t / \varepsilon}
\end{gathered}
$$

We used that $\frac{d}{d t}\left(-\alpha \varphi\left(\mathbf{u}_{\varepsilon}(\eta t)\right)+\Phi\left(\mathbf{u}_{\varepsilon}(\eta t), \varphi\left(\mathbf{u}_{\varepsilon}(\eta t)\right)\right)\right)=0$ and Assumption 4. Insertion of (80) into (78) yields

$$
\varepsilon \frac{d}{d t} \mathcal{V}_{\varepsilon}=-\left(\alpha-\frac{\partial \Phi^{\star}}{\partial v}\right) \mathcal{V}_{\varepsilon}+g_{1}(t)
$$

with $g_{1}(t):=-\varepsilon \frac{\nabla_{u} \Phi\left(\mathbf{u}_{\varepsilon}, \varphi\left(\mathbf{u}_{\varepsilon}\right)\right)}{\alpha-\frac{\partial \Phi}{\partial v}\left(\mathbf{u}_{\varepsilon}, \varphi\left(\mathbf{u}_{\varepsilon}\right)\right)} \cdot \mathbf{f}\left(\mathbf{u}_{\varepsilon}, v_{\varepsilon}\right)+I(t)$, satisfying $\left|g_{1}\right| \leq C\left(t e^{-M_{0} t / \varepsilon}+\varepsilon\right) \leq C \varepsilon$.

Assumption 4, Corollary 1 and Remark 3 imply $-\left(\alpha-\frac{\partial \Phi^{\star}}{\partial v}\right)<-M_{0}<0$.

Using Young's inequality we obtain

$$
\left|\mathcal{V}_{\varepsilon}(t)\right| \leq \int_{0}^{t} e^{-M_{0}(t-\tau) / \varepsilon} \frac{\left|g_{1}(\tau)\right|}{\varepsilon} d \tau \leq C \varepsilon
$$

\subsection{Proof of Proposition 2}

We obtain 


$$
\begin{gathered}
\frac{d \delta_{\varepsilon}}{d t}=\mathbf{f}\left(\mathbf{A}+\delta_{\varepsilon}, \varphi\left(\mathbf{A}+\delta_{\varepsilon}, t\right)+\zeta_{0}\left(\frac{t}{\varepsilon}\right)+\mathcal{V}_{\varepsilon}, t\right)-\mathbf{f}(\mathbf{A}, \varphi(\mathbf{A}, t), t) \\
\delta_{\varepsilon}(0)=0 .
\end{gathered}
$$

Due to Lipschitz continuity of the right hand side there exists a unique solution to this problem. It holds

$$
\begin{gathered}
\mathbf{f}\left(\mathbf{A}+\delta_{\varepsilon}, \varphi\left(\mathbf{A}+\delta_{\varepsilon}, t\right), t\right)-\mathbf{f}(\mathbf{A}, \varphi(\mathbf{A}, t), t)=\left(\nabla_{u} \mathbf{f}+\partial_{v} \mathbf{f} \otimes \nabla \varphi\right)(\eta(t)) \delta_{\varepsilon} \\
\left|\mathbf{f}\left(\mathbf{A}+\delta_{\varepsilon}, \varphi\left(\mathbf{A}+\delta_{\varepsilon}, t\right)+\zeta_{0}\left(\frac{t}{\varepsilon}\right)+\mathcal{V}_{\varepsilon}, t\right)-\mathbf{f}\left(\mathbf{A}+\delta_{\varepsilon}, \varphi\left(\mathbf{A}+\delta_{\varepsilon}, t\right), t\right)\right| \\
\leq C\left(e^{-M_{0} t / \varepsilon}+\varepsilon\right),
\end{gathered}
$$

where $\eta(t)$ is between $\mathbf{A}(t)$ and $\mathbf{A}(t)+\delta_{\varepsilon}(t)$. For the latter estimate we use Proposition 1 and (41). We obtain

$$
\frac{d \delta_{\varepsilon}}{d t}=\left(\nabla_{u} \mathbf{f}+\partial_{v} \mathbf{f} \otimes \nabla \varphi\right)(\eta(t)) \delta_{\varepsilon}+\mathbf{g}_{2}(t)
$$

with $\left|\mathbf{g}_{2}\right| \leq C\left(e^{-M_{0} t / \varepsilon}+\varepsilon\right)$. We note that if $\varepsilon$ is small enough, $\eta$ remains in a predefined vicinity of $\mathbf{A}$. Therefore, we can use the exponential dichotomy property from Remark 2 . Then Assumption 5 implies

$$
\left|\delta_{\varepsilon}\right| \leq C \varepsilon\left\|e^{-M t}\right\|_{L^{1}(\mathbb{R})} \leq C \varepsilon
$$

deltaep1s

\subsection{Proof of Remark 6}

We start from equation

$$
\frac{d \delta_{\varepsilon}}{d t}=\left(\nabla_{u} \mathbf{f}+\partial_{v} \mathbf{f} \otimes \nabla \varphi\right)(\eta(t)) \delta_{\varepsilon}+\mathbf{g}_{2}(t)
$$

It implies

$$
\frac{d\left|\delta_{\varepsilon}\right|}{d t}-\underbrace{\left(\nabla_{u} \mathbf{f}+\partial_{v} \mathbf{f} \otimes \nabla \varphi\right)(\eta(t)) \delta_{\varepsilon} \cdot \frac{\delta_{\varepsilon}}{\left|\delta_{\varepsilon}\right|}}_{\leq 0}=\mathbf{g}_{\mathbf{2}}(t) \cdot \operatorname{sign} \delta_{\varepsilon} .
$$

The integration of (87) yields estimate (59).

\subsection{Proof of Proposition 3}

We set $\xi(\mathbf{x}):=\alpha-\frac{\partial \Phi}{\partial v}(\mathbf{x}, \varphi(\mathbf{x}))$ and

$$
\mathcal{U}_{\varepsilon}=v_{\varepsilon}-\varphi\left(\mathbf{u}_{\varepsilon}\right)+\frac{\varepsilon}{\xi\left(\mathbf{u}_{\varepsilon}\right)} \frac{d}{d t} \varphi\left(\mathbf{u}_{\varepsilon}\right)-\zeta_{0}(t / \varepsilon)-\varepsilon \zeta_{1}(t / \varepsilon),
$$

where $\zeta_{0}$ is given by (40) and $\zeta_{1}$ by (62). 
Then, $\mathcal{U}_{\varepsilon}(0)=0$ and $\left|\zeta_{1}(t)\right| \leq C e^{-\alpha t}$. Using (40) and definition of $\varphi$ we obtain the following equation for $\mathcal{U}_{\varepsilon}$

$$
\begin{gathered}
\varepsilon \frac{d}{d t} \mathcal{U}_{\varepsilon}=-\alpha v_{\varepsilon}+\Phi\left(\mathbf{u}_{\varepsilon}, v_{\varepsilon}\right)-\varepsilon \frac{d}{d t} \varphi\left(\mathbf{u}_{\varepsilon}\right)+\varepsilon^{2} \frac{d}{d t}\left(\frac{\frac{d}{d t} \varphi\left(\mathbf{u}_{\varepsilon}\right)}{\xi\left(\mathbf{u}_{\varepsilon}\right)}\right) \\
-\varepsilon \frac{d}{d t} \zeta_{0}(t / \varepsilon)-\varepsilon^{2} \frac{d}{d t} \zeta_{1}(t / \varepsilon) \\
=-\alpha \mathcal{U}_{\varepsilon}-\alpha \varphi\left(\mathbf{u}_{\varepsilon}\right)-\alpha \zeta_{0}(t / \varepsilon)-\alpha \varepsilon \zeta_{1}(t / \varepsilon)+\varepsilon \frac{\frac{\partial \Phi}{\partial v}\left(\mathbf{u}_{\varepsilon}, \varphi\left(\mathbf{u}_{\varepsilon}\right)\right) \frac{d}{d t} \varphi\left(\mathbf{u}_{\varepsilon}\right)}{\xi\left(\mathbf{u}_{\varepsilon}\right)} \\
+\Phi\left(\mathbf{u}_{\varepsilon}, v_{\varepsilon}\right)+\varepsilon^{2} \frac{d}{d t}\left(\frac{\frac{d}{d t} \varphi\left(\mathbf{u}_{\varepsilon}\right)}{\xi\left(\mathbf{u}_{\varepsilon}\right)}\right)-\varepsilon \frac{d}{d t} \zeta_{0}(t / \varepsilon)-\varepsilon^{2} \frac{d}{d t} \zeta_{1}(t / \varepsilon) \\
=-\left(\alpha-\partial_{v} \Phi^{(1)}\right) \mathcal{U}_{\varepsilon}+\Phi\left(\mathbf{u}_{\varepsilon}, \varphi\left(\mathbf{u}_{\varepsilon}\right)-\frac{\frac{d}{d t} \varphi\left(\mathbf{u}_{\varepsilon}\right)}{\xi\left(\mathbf{u}_{\varepsilon}\right)} \varepsilon+\zeta_{0}(t / \varepsilon)+\varepsilon \zeta_{1}(t / \varepsilon)\right) \\
-\Phi\left(\mathbf{u}_{\varepsilon}, \varphi\left(\mathbf{u}_{\varepsilon}\right)\right)+\Phi\left(\mathbf{u}^{\mathbf{0}}, \varphi\left(\mathbf{u}^{\mathbf{0}}\right)\right)-\Phi\left(\mathbf{u}^{\mathbf{0}}, \varphi\left(\mathbf{u}^{\mathbf{0}}\right)+\zeta_{0}(t / \varepsilon)\right) \\
+\varepsilon^{2} \frac{d}{d t}\left(\frac{\frac{d}{d t} \varphi\left(\mathbf{u}_{\varepsilon}\right)}{\xi\left(\mathbf{u}_{\varepsilon}\right)}\right)+\varepsilon \frac{\partial_{v} \Phi\left(\mathbf{u}_{\varepsilon}, \varphi\left(\mathbf{u}_{\varepsilon}\right)\right)}{\xi\left(\mathbf{u}_{\varepsilon}\right)} \frac{d}{d t} \varphi\left(\mathbf{u}_{\varepsilon}\right) \\
=-\left(\alpha-\partial_{v} \Phi^{(1)}\right) \mathcal{U}_{\varepsilon}+G_{\varepsilon}+\varepsilon^{2} \frac{d}{d t}\left(\frac{1}{\xi\left(\mathbf{u}_{\varepsilon}\right)} \frac{d}{d t} \varphi\left(u_{\varepsilon}\right)\right) .
\end{gathered}
$$

Here, $\partial_{v} \Phi^{(1)}:=\partial_{v} \Phi\left(\mathbf{u}_{\varepsilon}, v^{(1)}\right)$ with $v^{(1)}$ between $\varphi\left(\mathbf{u}_{\varepsilon}\right)-\frac{\varepsilon}{\xi\left(\mathbf{u}_{\varepsilon}\right)} \frac{d}{d t} \varphi\left(\mathbf{u}_{\varepsilon}\right)+\zeta_{0}(t / \varepsilon)+\varepsilon \zeta_{1}(t / \varepsilon)$ and $\varphi\left(\mathbf{u}_{\varepsilon}\right)-\frac{\varepsilon}{\xi\left(\mathbf{u}_{\varepsilon}\right)} \frac{d}{d t} \varphi\left(\mathbf{u}_{\varepsilon}\right)+\zeta_{0}(t / \varepsilon)+\varepsilon \zeta_{1}(t / \varepsilon)+\mathcal{U}_{\varepsilon}$.

Next, we estimate the term $G_{\varepsilon}$.

$$
\begin{gathered}
\Phi\left(\mathbf{u}_{\varepsilon}, \varphi\left(\mathbf{u}_{\varepsilon}\right)-\frac{\varepsilon}{\xi\left(\mathbf{u}_{\varepsilon}\right)} \frac{d}{d t} \varphi\left(\mathbf{u}_{\varepsilon}\right)+\zeta_{0}(t / \varepsilon)+\varepsilon \zeta_{1}(t / \varepsilon)\right)-\Phi\left(\mathbf{u}_{\varepsilon}, \varphi\left(\mathbf{u}_{\varepsilon}\right)\right) \\
+\Phi\left(\mathbf{u}^{\mathbf{0}}, \varphi\left(\mathbf{u}^{\mathbf{0}}\right)\right)-\Phi\left(\mathbf{u}^{\mathbf{0}}, \varphi\left(\mathbf{u}^{\mathbf{0}}\right)+\zeta_{0}(t / \varepsilon)\right)+\varepsilon \frac{\partial_{v} \Phi\left(\mathbf{u}_{\varepsilon}, \varphi\left(\mathbf{u}_{\varepsilon}\right)\right)}{\xi\left(\mathbf{u}_{\varepsilon}\right)} \frac{d}{d t} \varphi\left(\mathbf{u}_{\varepsilon}\right) \\
=\Phi\left(\mathbf{u}^{\mathbf{0}}, \varphi\left(\mathbf{u}^{\mathbf{0}}\right)\right)-\Phi\left(\mathbf{u}^{\mathbf{0}}, \varphi\left(\mathbf{u}^{\mathbf{0}}\right)+\zeta_{0}(t / \varepsilon)\right)-\Phi\left(\mathbf{u}_{\varepsilon}, \varphi\left(\mathbf{u}_{\varepsilon}\right)\right) \\
+\Phi\left(\mathbf{u}_{\varepsilon}, \varphi\left(\mathbf{u}_{\varepsilon}\right)+\zeta_{0}(t / \varepsilon)+\varepsilon \zeta_{1}(t / \varepsilon)\right)+\partial_{v v} \Phi^{(2)} \frac{\varepsilon^{2}}{2\left(\xi\left(\mathbf{u}_{\varepsilon}\right)\right)^{2}}\left(\frac{d}{d t} \varphi\left(\mathbf{u}_{\varepsilon}\right)\right) \\
-\left(\partial_{v} \Phi\left(\mathbf{u}_{\varepsilon}, \varphi\left(\mathbf{u}_{\varepsilon}\right)+\zeta_{0}(t / \varepsilon)+\varepsilon \zeta_{1}(t / \varepsilon)\right)-\partial_{v} \Phi\left(\mathbf{u}_{\varepsilon}, \varphi\left(\mathbf{u}_{\varepsilon}\right)\right)\right) \frac{\varepsilon}{\xi\left(\mathbf{u}_{\varepsilon}\right)} \frac{d}{d t} \varphi\left(\mathbf{u}_{\varepsilon}\right),
\end{gathered}
$$

where $\partial_{v v} \Phi^{(2)}=\partial_{v v} \Phi\left(\mathbf{u}_{\varepsilon}, v^{(2)}\right)$ for an appropriate value $v^{(2)}$.

We start by estimating the two residuals in (89). Due to the boundedness of derivatives of $\Phi$ and $\mathbf{f}\left(\mathbf{u}_{\varepsilon}, v_{\varepsilon}\right)$, and exponential decay of the initial layers we obtain

$$
\begin{gathered}
\left|\left(\partial_{v} \Phi\left(\mathbf{u}_{\varepsilon}, \varphi\left(\mathbf{u}_{\varepsilon}\right)+\zeta_{0}(t / \varepsilon)+\varepsilon \zeta_{1}(t / \varepsilon)\right)-\partial_{v} \Phi\left(\mathbf{u}_{\varepsilon}, \varphi\left(\mathbf{u}_{\varepsilon}\right)\right)\right) \frac{\frac{d}{d t} \varphi\left(\mathbf{u}_{\varepsilon}\right)}{\xi\left(\mathbf{u}_{\varepsilon}\right)} \varepsilon\right| \\
+\left|\frac{\partial_{v v} \Phi^{(2)}}{2\left(\xi\left(\mathbf{u}_{\varepsilon}\right)\right)^{2}}\left(\frac{d}{d t} \varphi\left(\mathbf{u}_{\varepsilon}\right)\right) \varepsilon^{2}\right| \leq C\left(\varepsilon^{2}+\varepsilon e^{-\kappa t / \varepsilon}\right) .
\end{gathered}
$$


Estimating the remaining terms in (89) involves the boundary layers $\zeta_{0}$ and $\zeta_{1}$ and the use of the integral remainder for Taylor's formula:

$$
\begin{gathered}
\mid \Phi\left(\mathbf{u}_{\varepsilon}, \varphi\left(\mathbf{u}_{\varepsilon}\right)+\zeta_{0}(t / \varepsilon)+\varepsilon \zeta_{1}(t / \varepsilon)\right)-\Phi\left(\mathbf{u}_{\varepsilon}, \varphi\left(\mathbf{u}_{\varepsilon}\right)\right) \\
+\Phi\left(\mathbf{u}^{\mathbf{0}}, \varphi\left(\mathbf{u}^{\mathbf{0}}\right)\right)-\Phi\left(\mathbf{u}^{\mathbf{0}}, \varphi\left(\mathbf{u}^{\mathbf{0}}\right)+\zeta_{0}(t / \varepsilon)\right) \mid \\
=\left|\int_{0}^{1}\left(\mathbf{u}_{\varepsilon}-\mathbf{u}^{\mathbf{0}}\right) \cdot \nabla_{x} \Phi\left(\mathbf{x}, \varphi(\mathbf{x})+\zeta_{0}(t / \varepsilon)\right)\right|_{\mathbf{x}=\mathbf{u}^{\mathbf{0}}+\eta\left(\mathbf{u}_{\varepsilon}-\mathbf{u}^{\mathbf{0}}\right)} d \eta \\
-\left.\int_{0}^{1}\left(\mathbf{u}_{\varepsilon}-\mathbf{u}^{\mathbf{0}}\right) \cdot \nabla_{x} \Phi(\mathbf{x}, \varphi(\mathbf{x}))\right|_{\mathbf{x}=\mathbf{u}^{\mathbf{0}}+\eta\left(\mathbf{u}_{\varepsilon}-\mathbf{u}^{\mathbf{0}}\right)} d \eta \mid+C \varepsilon e^{-\kappa t / \varepsilon} \\
\leq t \sum_{i=1}^{m}\left\|\frac{d}{d t} u_{\varepsilon}^{i}\right\|_{\infty}\left\|\partial_{v} \nabla_{x^{i}} \Phi(\mathbf{x}, \varphi(\mathbf{x}))\right\|_{\infty} C e^{-\kappa t / \varepsilon}+C \varepsilon e^{-\kappa t / \varepsilon} \\
\leq t C e^{-\kappa t / \varepsilon}+C \varepsilon e^{-\kappa t / \varepsilon} .
\end{gathered}
$$

It remains to estimate the term $\varepsilon^{2} \frac{d}{d t}\left(\frac{1}{\xi\left(\mathbf{u}_{\varepsilon}\right)} \frac{d}{d t} \varphi\left(u_{\varepsilon}\right)\right)$ in (88). We start by the estimate for $\frac{d}{d t} v_{\varepsilon}$. Theorem 1 and the equation $\alpha \varphi(\mathbf{A})=\Phi(\mathbf{A}, \varphi(\mathbf{A}))$ yield

$$
\begin{aligned}
\left|\frac{d}{d t} v_{\varepsilon}(t)\right|=\frac{\alpha}{\varepsilon} \mid-v_{\varepsilon}(t)+ & \varphi(\mathbf{A}(t))-\frac{\Phi(\mathbf{A}(t), \varphi(\mathbf{A}(t)))}{\alpha}+\frac{\Phi\left(\mathbf{u}_{\varepsilon}(t), v_{\varepsilon}(t)\right)}{\alpha} \mid \\
& \leq C\left(1+\frac{1}{\varepsilon} e^{-M_{0} t / \varepsilon}\right) .
\end{aligned}
$$

Using $\nabla \varphi(x)=\frac{\nabla_{u} \Phi(x, \varphi(x))}{\xi(x)}$, we obtain

$$
\begin{aligned}
g_{4}(t):=\frac{d}{d t} & \left(\frac{1}{\xi\left(\mathbf{u}_{\varepsilon}\right)} \frac{d}{d t} \varphi\left(\mathbf{u}_{\varepsilon}\right)\right)=\frac{d}{d t}\left(\frac{\nabla \Phi\left(\mathbf{u}_{\varepsilon}, \varphi\left(\mathbf{u}_{\varepsilon}\right)\right) \cdot \mathbf{f}\left(\mathbf{u}_{\varepsilon}, v_{\varepsilon}\right)}{\left(\xi\left(\mathbf{u}_{\varepsilon}\right)\right)^{2}}\right) \\
= & \nabla_{u}\left(\frac{\nabla \Phi\left(\mathbf{u}_{\varepsilon}, \varphi\left(\mathbf{u}_{\varepsilon}\right)\right) \cdot \mathbf{f}\left(\mathbf{u}_{\varepsilon}, v_{\varepsilon}\right)}{\left(\xi\left(\mathbf{u}_{\varepsilon}\right)\right)^{2}}\right) \cdot \mathbf{f}\left(\mathbf{u}_{\varepsilon}, \mathbf{v}_{\varepsilon}\right) \\
& +\frac{\partial}{\partial v}\left(\frac{\nabla \Phi\left(\mathbf{u}_{\varepsilon}, \varphi\left(\mathbf{u}_{\varepsilon}\right)\right) \cdot \mathbf{f}\left(\mathbf{u}_{\varepsilon}, v_{\varepsilon}\right)}{\left(\xi\left(\mathbf{u}_{\varepsilon}\right)\right)^{2}}\right) \frac{d}{d t} v_{\varepsilon} .
\end{aligned}
$$

Due to the boundedness of derivatives of $\mathbf{f}$ and $\Phi$ and equation (92) it follows

$$
\left|g_{4}(t)\right| \leq C\left(1+\frac{1}{\varepsilon} e^{-M_{0} t / \varepsilon}\right)
$$

Inserting equations (90) and (91) in equation (89), and finally equation (89) and estimate (94) in equation (88), we obtain

$$
\varepsilon \frac{d}{d t} \mathcal{U}_{\varepsilon}=-\left(\alpha-\partial_{v} \Phi^{(1)}\right) \mathcal{U}_{\varepsilon}+g_{5}(t)
$$

with $\left|g_{5}(t)\right| \leq C\left(\varepsilon^{2}+(\varepsilon+t) e^{-\kappa t / \varepsilon}\right)$. 
Using the explicit formula for $\mathcal{U}_{\varepsilon}$ and Young's inequality yields

$$
\begin{gathered}
\left|\mathcal{U}_{\varepsilon}(t)\right| \leq C \int_{0}^{t} e^{-M_{0}(t-\tau) / \varepsilon}\left(\varepsilon+\left(1+\frac{\tau}{\varepsilon}\right) e^{-\kappa \tau / \varepsilon}\right) d \tau \\
=C \int_{0}^{t} e^{-M_{0}(t-\tau) / \varepsilon} \varepsilon d \tau+C \int_{0}^{t} e^{-M_{0}(t-\tau) / \varepsilon}\left(1+\frac{\tau}{\varepsilon}\right) e^{-\kappa \tau / \varepsilon} d \tau \\
=: \mathcal{U}_{\varepsilon, 1}+\mathcal{U}_{\varepsilon, 2},
\end{gathered}
$$

where

$$
\left\|\mathcal{U}_{\varepsilon, 1}\right\|_{L^{\infty}\left(\mathbb{R}_{+}\right)} \leq C \varepsilon^{2}, \quad\left\|\mathcal{U}_{\varepsilon, 2}\right\|_{L^{1}\left(\mathbb{R}_{+}\right)} \leq C \varepsilon^{2}
$$

mathcalU

\subsection{Proof of Proposition 4}

As above, we set $\xi(\mathbf{x}):=\alpha-\frac{\partial \Phi}{\partial v}(\mathbf{x}, \varphi(\mathbf{x}))$. Adopting the notations from Proposition 3 , we obtain

$$
\frac{d}{d t} \mathbf{u}_{\varepsilon}=\mathbf{f}\left(\mathbf{u}, \mathcal{U}_{\varepsilon}+\varphi\left(\mathbf{u}_{\varepsilon}\right)-\frac{\frac{d}{d t} \varphi\left(\mathbf{u}_{\varepsilon}\right)}{\xi\left(\mathbf{u}_{\varepsilon}\right)} \varepsilon+\zeta_{0}(t / \varepsilon)+\varepsilon \zeta_{1}(t / \varepsilon)\right) .
$$

In order to obtain an $\mathcal{O}\left(\varepsilon^{2}\right)$ approximation, we expand $\mathbf{u}_{\varepsilon}$ as

$$
\mathbf{u}_{\varepsilon}=\mathbf{A}+\varepsilon\left(\mathbf{u}^{1}(t)+\psi^{1}(t / \varepsilon)\right)+\ldots
$$

Then it is required that $\mathbf{u}^{1}(0)+\psi^{1}(0)=0$. We obtain

$$
\left\{\begin{array}{l}
\frac{d}{d \tau} \psi^{1}=\mathbf{f}\left(\mathbf{u}^{0}, \varphi\left(\mathbf{u}^{0}\right)+\zeta_{0}(\tau)\right)-\mathbf{f}\left(\mathbf{u}^{0}, \varphi\left(\mathbf{u}^{0}\right)\right) \\
\psi^{1}(0)=-\int_{0}^{\infty} \mathbf{f}\left(\mathbf{u}^{0}, \varphi\left(\mathbf{u}^{0}\right)+\zeta_{0}(\tau)\right)-\mathbf{f}\left(\mathbf{u}^{0}, \varphi\left(\mathbf{u}^{0}\right)\right) d \tau
\end{array}\right.
$$

We recall that $\mathbf{u}^{1}$ satisfies

$$
\left\{\begin{array}{l}
\frac{d}{d t} \mathbf{u}^{1}=\left(\nabla_{u} \mathbf{f}(\mathbf{u}, v)+\partial_{v} \mathbf{f}(\mathbf{u}, f) \otimes \nabla \varphi(\mathbf{u})\right)_{\mathbf{u}=\mathbf{A}, v=\varphi(\mathbf{A})} \mathbf{u}^{1}-\partial_{v} \mathbf{f}^{A} \frac{\nabla \varphi(\mathbf{A}) \cdot \mathbf{f}^{A}}{\xi(\mathbf{A})} \\
\mathbf{u}^{1}(0)=-\psi^{1}(0),
\end{array}\right.
$$

where $\mathbf{f}^{A}:=\mathbf{f}(\mathbf{A}, \varphi(\mathbf{A}))$ and $\partial_{v} \mathbf{f}^{A}:=\left.\partial_{v} \mathbf{f}(\mathbf{u}, \mathbf{v})\right|_{\mathbf{u}=\mathbf{A}, v=\varphi(\mathbf{A})}$ etc.

In the following we use the notation

$$
D_{u} f^{A}:=\left(\nabla_{u} \mathbf{f}(\mathbf{u}, v)+\partial_{v} \mathbf{f}(\mathbf{u}, v) \otimes \nabla \varphi(\mathbf{u})\right)_{\mathbf{u}=\mathbf{A}, v=\varphi(\mathbf{A})} .
$$

Note that according to Assumptions 3 and $5 \mathbf{u}^{1}$ is bounded.

We now estimate the approximation error

$$
\hat{\delta}_{\varepsilon}(t)=\mathbf{u}_{\varepsilon}(t)-\mathbf{A}(t)-\varepsilon \mathbf{u}^{1}(t)-\varepsilon \psi^{1}(t / \varepsilon) .
$$


We obtain

$$
\begin{gathered}
\frac{d}{d t} \hat{\delta}_{\varepsilon}=\mathbf{f}\left(\mathbf{A}+\varepsilon \mathbf{u}^{1}+\varepsilon \psi^{1}(t / \varepsilon)+\hat{\delta}_{\varepsilon}, \mathcal{U}_{\varepsilon}\right. \\
+\varphi\left(\mathbf{A}(t)+\varepsilon \mathbf{u}^{1}(t)+\varepsilon \psi^{1}(t / \varepsilon)+\hat{\delta}_{\varepsilon}\right)-\varepsilon \frac{\nabla \varphi\left(\mathbf{u}_{\varepsilon}\right) \cdot \mathbf{f}\left(\mathbf{u}_{\varepsilon}, v_{\varepsilon}\right)}{\xi\left(\mathbf{u}_{\varepsilon}\right)} \\
\left.+\varepsilon \frac{\nabla \varphi(\mathbf{A}) \cdot \frac{d}{d t} \mathbf{A}}{\xi(\mathbf{A})}-\varepsilon \frac{\nabla \varphi(\mathbf{A}) \cdot \frac{d}{d t} \mathbf{A}}{\xi(\mathbf{A})}+\zeta_{0}(t / \varepsilon)+\varepsilon \zeta_{1}(t / \varepsilon)\right) \\
-\frac{d}{d t} \mathbf{u}^{1} \varepsilon-\varepsilon \frac{d}{d t} \psi^{1}(t / \varepsilon)-\mathbf{f}(\mathbf{A}, \varphi(\mathbf{A})) \\
=\mathbf{f}\left(\mathbf{A}+\varepsilon \mathbf{u}^{1},-\varepsilon \frac{\nabla \varphi(\mathbf{A}) \cdot \frac{d}{d t} \mathbf{A}}{\xi(\mathbf{A})}+\zeta_{0}(t / \varepsilon)+\varphi\left(\mathbf{A}+\varepsilon \mathbf{u}^{1}\right)\right) \\
+D_{u} \hat{\mathbf{f}}^{*}\left(\varepsilon \psi^{1}(t / \varepsilon)+\hat{\delta}_{\varepsilon}\right)-\frac{d}{d t} \mathbf{u}^{1} \varepsilon-\varepsilon \frac{d}{d t} \psi^{1}(t / \varepsilon)-\mathbf{f}(\mathbf{A}, \varphi(\mathbf{A})) \\
+\partial_{v} \mathbf{f}^{*}\left(\mathcal{U}_{\varepsilon}-\varepsilon \frac{\nabla \varphi\left(\mathbf{u}_{\varepsilon}\right) \cdot \mathbf{f}\left(\mathbf{u}_{\varepsilon}, v_{\varepsilon}\right)}{\xi\left(\mathbf{u}_{\varepsilon}\right)}+\varepsilon \frac{\nabla \varphi(\mathbf{A}) \cdot \frac{d}{d t} \mathbf{A}}{\xi(\mathbf{A})}+\varepsilon \zeta_{1}(t / \varepsilon)\right)
\end{gathered}
$$

where $\partial_{v} \mathbf{f}^{*}=\partial_{v} \mathbf{f}\left(\mathbf{u}_{\varepsilon}, v^{*}\right)$ for an appropriate value $v^{*}$ and

$$
\begin{gathered}
D_{u} \hat{\mathbf{f}}^{*}=\left.\nabla_{u} \mathbf{f}\left(\mathbf{x}, \varphi(\mathbf{x})-\frac{\varepsilon}{\xi(\mathbf{A})} \nabla \varphi(\mathbf{A}) \cdot \frac{d}{d t} \mathbf{A}+\zeta_{0}(t / \varepsilon)\right)\right|_{\mathbf{x}=\mathbf{u} *} \\
+\left.\partial_{v} \mathbf{f}\left(\mathbf{x}, \varphi(\mathbf{x})-\frac{\varepsilon}{\xi(\mathbf{A})} \nabla \varphi(\mathbf{A}) \cdot \frac{d}{d t} \mathbf{A}+\zeta_{0}(t / \varepsilon)\right) \otimes \nabla \varphi(\mathbf{x})\right|_{\mathbf{x}=\mathbf{u} *}
\end{gathered}
$$

for an appropriate value $\mathbf{u}^{*}$ between $\mathbf{u}_{\varepsilon}$ and $\mathbf{A}+\varepsilon \mathbf{u}^{1}$.

The perturbation $\frac{\varepsilon}{\xi(\mathbf{A})} \nabla \varphi(\mathbf{A}) \cdot \frac{d}{d t} \mathbf{A}+\zeta_{0}(t / \varepsilon)$ tends to zero for $\varepsilon$ tending to zero and $t \geq \mathcal{O}(1)$. Therefore, $D_{u} \hat{\mathbf{f}}^{*}$ satisfies the exponential dichotomy from Assumption 5 .

Now we estimate the last term in (103):

$$
\begin{gathered}
\mathcal{U}_{\varepsilon}-\varepsilon \frac{\nabla \varphi\left(\mathbf{u}_{\varepsilon}\right) \cdot \mathbf{f}\left(\mathbf{u}_{\varepsilon}, v_{\varepsilon}\right)}{\xi\left(\mathbf{u}_{\varepsilon}\right)}+\varepsilon \frac{\nabla \varphi(\mathbf{A}) \cdot \frac{d}{d t} \mathbf{A}}{\xi(\mathbf{A})} \\
=\mathcal{U}_{\varepsilon}-\varepsilon \frac{\nabla \varphi\left(\mathbf{u}_{\varepsilon}\right) \cdot \mathbf{f}\left(\mathbf{u}_{\varepsilon}, v_{\varepsilon}\right)}{\xi\left(\mathbf{u}_{\varepsilon}\right)}+\left(\frac{\nabla \varphi(\mathbf{A}) \cdot \mathbf{f}(\mathbf{A}, \varphi(\mathbf{A}))}{\xi(\mathbf{A})}-\frac{\nabla \varphi\left(\mathbf{u}_{\varepsilon}\right) \cdot f\left(\mathbf{u}_{\varepsilon}, \varphi\left(\mathbf{u}_{\varepsilon}\right)\right)}{\xi\left(\mathbf{u}_{\varepsilon}\right)}\right) \varepsilon \\
+\frac{\nabla \varphi\left(\mathbf{u}_{\varepsilon}\right) \cdot f\left(\mathbf{u}_{\varepsilon}, \varphi\left(\mathbf{u}_{\varepsilon}\right)\right)}{\xi\left(\mathbf{u}_{\varepsilon}\right)} \varepsilon \\
=\hat{\mathcal{U}}_{\varepsilon}+\left(\frac{\nabla \varphi(\mathbf{A}) \cdot \mathbf{f}(\mathbf{A}, \varphi(\mathbf{A}))}{\xi(\mathbf{A})}-\frac{\nabla \varphi\left(\mathbf{u}_{\varepsilon}\right) \cdot f\left(\mathbf{u}_{\varepsilon}, \varphi\left(\mathbf{u}_{\varepsilon}\right)\right)}{\xi\left(\mathbf{u}_{\varepsilon}\right)}\right) \varepsilon
\end{gathered}
$$

remainder

with

$$
\hat{\mathcal{U}}_{\varepsilon}:=\mathcal{U}_{\varepsilon}-\varepsilon \frac{\nabla \varphi\left(\mathbf{u}_{\varepsilon}\right)}{\xi\left(\mathbf{u}_{\varepsilon}\right)} \cdot\left(\mathbf{f}\left(\mathbf{u}_{\varepsilon}, v_{\varepsilon}\right)-\mathbf{f}\left(\mathbf{u}_{\varepsilon}, \varphi\left(\mathbf{u}_{\varepsilon}\right)\right)\right)
$$


Since

$$
\left|\hat{\mathcal{U}}_{\varepsilon}\right| \leq \mathcal{U}_{\varepsilon}+\varepsilon C\left|\mathcal{V}_{\varepsilon}+\zeta_{0}(t / \varepsilon)\right|,
$$

using a similar argument as in (97), we obtain

$$
\hat{\mathcal{U}}_{\varepsilon}=: \hat{\mathcal{U}}_{\varepsilon, 1}+\hat{\mathcal{U}}_{\varepsilon, 2}
$$

with

$$
\left\|\hat{\mathcal{U}}_{\varepsilon, 1}\right\|_{L^{\infty}\left(\mathbb{R}_{+}\right)} \leq C \varepsilon^{2}, \quad\left\|\hat{\mathcal{U}}_{\varepsilon, 2}\right\|_{L^{1}\left(\mathbb{R}_{+}\right)} \leq C \varepsilon^{2}
$$

Next, Theorem 1 implies

$$
\left|\left(\frac{\nabla \varphi(\mathbf{A}) \cdot \mathbf{f}(\mathbf{A}, \varphi(\mathbf{A}))}{\xi(\mathbf{A})}-\frac{\nabla \varphi\left(\mathbf{u}_{\varepsilon}\right) \cdot f\left(\mathbf{u}_{\varepsilon}, \varphi\left(\mathbf{u}_{\varepsilon}\right)\right)}{\xi\left(\mathbf{u}_{\varepsilon}\right)}\right)\right| \leq C \varepsilon
$$

Insertion of (109) and (107) into (104) and insertion of (104) into equation (103) yield

$$
\begin{aligned}
\frac{d}{d t} \hat{\delta}_{\varepsilon} & =\mathbf{f}\left(\mathbf{A}+\varepsilon \mathbf{u}^{1},-\varepsilon \frac{\nabla \varphi(\mathbf{A}) \cdot \frac{d}{d t} \mathbf{A}}{\xi(\mathbf{A})}+\zeta_{0}(t / \varepsilon)+\varphi\left(\mathbf{A}+\varepsilon \mathbf{u}^{1}\right)\right)+D_{u} \hat{\mathbf{f}}^{*} \hat{\delta}_{\varepsilon} \\
& -\frac{d}{d t} \mathbf{u}^{1} \varepsilon-\varepsilon \frac{d}{d t} \psi^{1}(t / \varepsilon)-\mathbf{f}(\mathbf{A}, \varphi(\mathbf{A}))+\partial_{v} \mathbf{f}^{*} \hat{\mathcal{U}}_{\varepsilon, 2}+\varepsilon\left(\mathbf{G}_{1}+\mathbf{G}_{2}\right) .
\end{aligned}
$$

delatu20I

Using Assumption 5 we obtain the estimates $\left\|\mathbf{G}_{1}\right\|_{L^{\infty}\left(\mathbb{R}^{+}\right)} \leq C \varepsilon$ and $\left|\mathbf{G}_{2}(t)\right| \leq C e^{-M_{0} t / \varepsilon}$.

Using boundedness of $\mathbf{u}^{1}$, we calculate

$$
\begin{gathered}
\mathbf{f}\left(\mathbf{A}+\varepsilon \mathbf{u}^{1},-\varepsilon \frac{\nabla \varphi(\mathbf{A}) \cdot \frac{d}{d t} \mathbf{A}}{\xi(\mathbf{A})}+\zeta_{0}(t / \varepsilon)+\varphi\left(\mathbf{A}+\varepsilon \mathbf{u}^{1}\right)\right) \\
=\mathbf{f}\left(\mathbf{A}, \zeta_{0}(t / \varepsilon)+\varphi(\mathbf{A})\right)+D_{u} \mathbf{f}^{A} \varepsilon \mathbf{u}^{1}-\partial_{v} \mathbf{f}^{A} \frac{\nabla \varphi(\mathbf{A}) \cdot \frac{d}{d t} \mathbf{A}}{\xi(\mathbf{A})} \varepsilon \\
+\varepsilon\left(\mathbf{G}_{3}+\mathbf{G}_{4}\right)
\end{gathered}
$$

with $\left\|\mathbf{G}_{3}\right\|_{L^{\infty}\left(\mathbb{R}^{+}\right)} \leq C \varepsilon$ and $\left|\mathbf{G}_{4}(t)\right| \leq C e^{-M_{0} t / \varepsilon}$.

Insertion of equation (111) into equation (110) for $\hat{\delta}_{\varepsilon}$ leads to

$$
\begin{aligned}
\frac{d}{d t} \hat{\delta}_{\varepsilon}= & \mathbf{f}\left(\mathbf{A}, \zeta_{0}(t / \varepsilon)+\varphi(\mathbf{A})\right)-\mathbf{f}(\mathbf{A}, \varphi(\mathbf{A}))+D_{u} \hat{\mathbf{f}}^{*} \hat{\delta}_{\varepsilon} \\
+ & {\left[D_{u} \mathbf{f}^{A} u^{1}-\partial_{v} \mathbf{f}^{A} \frac{\nabla \varphi(\mathbf{A}) \cdot \frac{d}{d t} \mathbf{A}}{\xi(\mathbf{A})} \varepsilon-\frac{d}{d t} \mathbf{u}^{1}\right] \varepsilon } \\
& -\varepsilon \frac{d}{d t} \psi^{1}(t / \varepsilon)+\partial_{v} \mathbf{f}^{*} \hat{\mathcal{U}}_{\varepsilon, 2}+\varepsilon\left(\mathbf{G}_{5}+\mathbf{G}_{6}\right)
\end{aligned}
$$

with $\left\|\mathbf{G}_{5}\right\|_{L^{\infty}\left(\mathbb{R}^{+}\right)} \leq C \varepsilon$ and $\left|\mathbf{G}_{6}(t)\right| \leq C e^{-M_{0} t / \varepsilon}$. 
Finally, insertion of equations (101) and (100) into (112) leads to

$$
\begin{gathered}
\frac{d}{d t} \hat{\delta}_{\varepsilon}=\mathbf{f}\left(\mathbf{A}, \zeta_{0}(t / \varepsilon)+\varphi(\mathbf{A})\right)-\mathbf{f}(\mathbf{A}, \varphi(\mathbf{A}))+D_{u} \hat{\mathbf{f}}^{*} \hat{\delta}_{\varepsilon} \\
-\mathbf{f}\left(\mathbf{u}^{0}, \varphi\left(\mathbf{u}^{0}\right)+\zeta_{0}(t / \varepsilon)\right)+\mathbf{f}\left(\mathbf{u}^{0}, \varphi\left(\mathbf{u}^{0}\right)\right)+\varepsilon\left(\mathbf{G}_{5}+\mathbf{G}_{6}\right)+\partial_{v} \mathbf{f}^{*} \hat{\mathcal{U}}_{\varepsilon, 2} .
\end{gathered}
$$

A precise Taylor estimate results in

$$
\begin{gathered}
\left\|\mathbf{f}\left(\mathbf{A}, \zeta_{0}(t / \varepsilon)+\varphi(\mathbf{A})\right)-\mathbf{f}(\mathbf{A}, \varphi(\mathbf{A}))-\mathbf{f}\left(\mathbf{u}^{0}, \varphi\left(\mathbf{u}^{0}\right)+\zeta_{0}(t / \varepsilon)\right)+\mathbf{f}\left(\mathbf{u}^{0}, \varphi\left(\mathbf{u}^{0}\right)\right)\right\|_{\infty} \\
=\left\|\int_{0}^{1}\left(\left.\nabla_{u} \mathbf{f}\left(\mathbf{u}, \varphi(\mathbf{u})+\zeta_{0}(t / \varepsilon)\right)\right|_{\mathbf{u}=\mathbf{u}^{0}+\nu\left(\mathbf{A}-\mathbf{u}^{0}\right)}-\left.D_{u} \mathbf{f}(\mathbf{u}, \varphi(\mathbf{u}))\right|_{\mathbf{u}=\mathbf{u}^{0}+\nu\left(\mathbf{A}-\mathbf{u}^{0}\right)}\right)\left(\mathbf{A}-\mathbf{u}^{0}\right) d \nu\right\|_{\infty} \\
\leq C \max \left\{\varepsilon^{2}, t e^{-M_{0} t / \varepsilon}\right\}\left\|\frac{d}{d t} \mathbf{A}\right\|_{\infty}\left\|\partial_{v} D_{u} \mathbf{f}(\mathbf{u}, \varphi(\mathbf{u})+v)\right\|_{\infty}=: G_{7} \max \left\{\varepsilon^{2}, t e^{-M_{0} t / \varepsilon}\right\} .
\end{gathered}
$$

In the final step we use estimate (114) and the exponential dichotomy for $D_{u}$, to obtain the inequality

$$
\begin{gathered}
\left|\hat{\delta}_{\varepsilon}\right| \leq \int_{0}^{t} e^{-M(t-\tau)}\left(\left|\varepsilon \mathbf{G}_{5}(\tau)+\varepsilon \mathbf{G}_{6}(\tau)+\partial_{v} \mathbf{f}^{*} \hat{\mathcal{U}}_{\varepsilon, 2}\right|+\tau e^{-M_{0} \tau / \varepsilon} G_{7}\right) d \tau \\
\leq C \varepsilon^{2}+C|| \hat{\mathcal{U}}_{\varepsilon, 2} \|_{L^{1}\left(\mathbb{R}_{+}\right)} \leq C \varepsilon^{2}
\end{gathered}
$$

\section{Acknowledgements}

AM-C and TS are supported by the Collaborative Research Center, SFB 873 "Maintenance and Differentiation of Stem Cells in Development and Disease" from the German Research Council (DFG). Research visits of AM at the Heidelberg University were supported by the Romberg Professorship of the Heidelberg Graduate School, Interdisciplinary Center for Scientific Computing (IWR), Heidelberg University.

\section{References}

Agarwal [1] R. P. Agarwal, D. O' Regan, An Introduction to Ordinary Differential Equations, Springer, Heidelberg, 2008.

BanLach [2] J. Banasiak, M. Lachowicz, Methods of small parameter in mathematical biology. Birkhäuser, Cham, 2014.

carr [3] J. Carr, Applications of Centre Manifold Theory, Springer, New York, 1981.

CGOPRL [4] L. Y. Chen, N. Goldenfeld, Y. Oono, Renormalization group theory for global asymptotic analysis, Phys. Rev. Lett. 73 (1994), 1311-1315

CGO:95 [5] L. Y. Chen, N .Goldenfeld, Y. Oono, Renormalization group and singular perturbations: Multiple scales, boundary layers, and reductive perturbation theory, Phys. Rev. E 54, (1996), 376-394.

Chiba:08

[6] H. Chiba, $C^{1}$ approximation of vector fields based on the renormalization group method, SIAM J. Appl. Dym. Syst., 7 (2008), 895-932. 
[7] H. Chiba, Approximation of Center Manifolds on the Renormalization Group Method, J. Math. Phys. 49, 102703 (2008).

[8] H. Chiba, Analysis of a renormalization group method and normal form theory for perturbed ordinary differential equations. Physica D: Nonlinear Phenomena 237 (2008), 1029-1052.

Chiba09 [9] H. Chiba, Extension and Unification of Singular Perturbation Methods for ODEs Based on the Renormalization Group Method, SIAM J. Appl. Dym. Syst. 8 (2009), 1066-1115.

[10] R. E. Lee DeVille, A. Harkin, M. Holzer, K. Josić, T. Kaper, Analysis of a renormalization group method and normal form theory for perturbed ordinary differential equations, Physica D, 237 (2008), 1029-1052.

Getto [11] P. Getto, A. Marciniak-Czochra, Y. Nakata, M. Vivanco, Global dynamics of twocompartment models for cell production systems with regulatory mechanisms, Math. biosci. 245 (2013), 258-268.

[12] F. Hoppensteadt, Singular perturbations on the infinite interval, Trans. Amer. Math. Soc., 123 (1966), 521-535.

Hopp71 [13] F. Hoppensteadt, Properties of Solutions of Ordinary Differential Equations with Small Parameters, Comm. Pure Appl. Math. 24 (1971), 807-840.

Hopp00 [14] F. Hoppensteadt, Analysis and Simulation of Chaotic Systems, 2nd edition, Applied Mathematical Sciences 94, Springer, 2000.

[15] T.J. Kaper, An introduction to geometric methods and dynamical systems theory for singular perturbation problems. analyzing multiscale phenomena using singular perturbation methods. In J. Cronin and R.E. OMalley, editors, Analyzing Multiscale Phenomena Using Singular Perturbation Methods, pages 85-131. Springer, 1999.

[16] Z. Lin, Y.-X. Lin, Linear Systems Exponential Dichotomy and Structure of Sets of Hyperbolic Points, World Scientific, Singapore, 2000.

[17] R. E. O'Malley, Jr., Singular perturbation methods for ordinary differential equations, Applied mathematical sciences 89, Springer, New York, Berlin, Heidelberg, 1991.

[18] R. E. O’Malley, Jr., E. Kirkinis, Studies in Applied Mathematics, 124 (2010), 383-410.

[19] A. Marciniak-Czochra, T. Stiehl, A. D. Ho, W. Jaeger, W. Wagner, Modeling asymmetric cell division in hematopietic stem cells regulation of self-renewal is essential for efficient repopulation, Stem Cells Dev. 18 (2009), 377-385.

[20] A. Marciniak-Czochra, A. Mikelić, Shadow limits via the renormalization group method and the center manifold method. Vietnam J. Math, 2016, DOI: 10.1007/s10013-016-0199-6.

MudaM [21] B. Mudavanhu and R. E. O'Malley, Jr., A New Renormalization Method for the Asymptotic Solution of Weakly Nonlinear Vector Systems, SIAM J. Appl. Math., 63(2) (2003), 373397. Equations, Journal of Dynamics and Differential Equations, 13, (2001), pp 275-321. 
Nakata [23] Y. Nakata, P. Getto, A. Marciniak-Czochra, T. Alarcon, Stability analysis of multicompartment models for cell production systems, J. Biol. Dyn. Vol 6. Suppl. 1 (2012), $2-18$.

[24] T. Stiehl, A. Marciniak-Czochra, Characterization of stem cells using mathematical models of multistage cell lineages, Math. Comp. Models. Vol 53 (2011) 1505-1517.

TemWir [25] R. Temam, D. Wirosoetisno, On the solutions of the renormalized equations at all orders, Adv. Diff. Eqn., 8 (2003), 1005-1024.

Tikh:85 [26] A.N. Tikhonov, A.B. Vasileva, A.G. Sveshnikov, Differential equations, (Translation of: Differentsial'nye uravneniiya), Springer-Verlag, Berlin, 1985.

Vasileva:93 [27] A. B. Vasil'eva, V. F. Butuzov, L. V. Kalachev, The Boundary Function Method for Singular Perturbed Problems, SIAM, Philadelphia, 1993.

Ziane1 [28] M. Ziane. On a certain renormalization group method, J. Math. Phys., 41(2000), 3290-3299. 